\title{
ON CERTAIN PERIODIC PROPERTIES OF CYCLIC COMPOSITIONS OF NUMBERS
}

\author{
By D. M. Y. SoMMERviLLE.
}

Communicated by Prof. W. Burnside.

[Received October 9th, 1908.-Read November 12th, 1908.-Received, in revised form, February 4th, 1909.]

$\S \S 1-3$. Introductory.

\section{CONTENTS.}

1. Statement of the problem. Row of order $N$ with $s$ symbols; evolution and involution; periodicity. Correspondence between pairs of symbols and single symbols; representation by multiplication table, its arbitrary

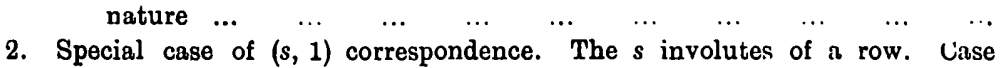
$\begin{array}{llllllll}\text { where the symbols form a group } & \ldots & \ldots & \ldots & \ldots & \ldots & \ldots\end{array}$

3. Order of the involutes in the case where the symbols form an Abelian group, and condition that an involute may be of the same order $\quad \ldots \quad 265$

I.

Elaboration of the Theory for Two Symbols.

4. Notation. Partitions, compositions and cyclic compositions. MIulti$\begin{array}{llllllllll}\text { partite numbers } & \ldots & \ldots & \ldots & \ldots & \ldots & \ldots & \ldots & \ldots & 268\end{array}$

5. Rules for evolution. Notation. Alternate rows $\quad \ldots \quad$...

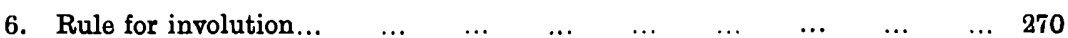

7. Definitions of proper, improper, regular, alien, degenerate and irreducible $\begin{array}{lllllllll}\text { rows or compositions } \ldots & \ldots & \ldots & \ldots & \ldots & \ldots & \ldots & \ldots & 270\end{array}$

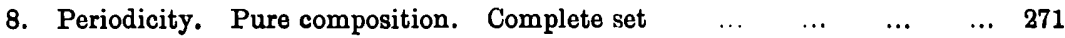

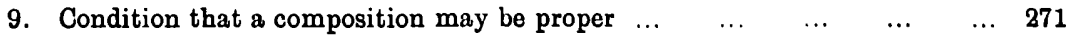

10. Symmetrical and enantiomorphic compositions and periods. Inverse and $\begin{array}{lllllllll}\text { self-inverse compositions } & \ldots & \ldots & \ldots & \ldots & \ldots & \ldots & \ldots & 272\end{array}$

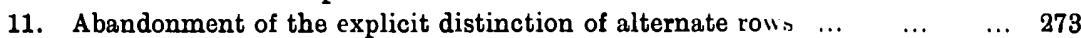

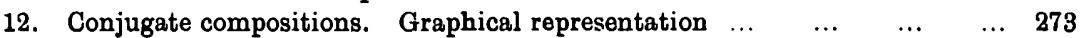

13. Reduction of a cyclic composition of $2 N$, modulo $N$. Interpretation of

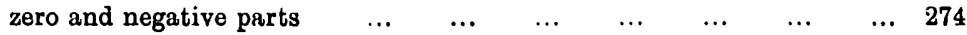

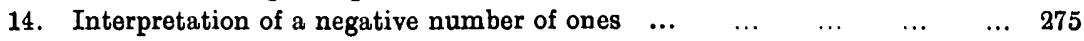

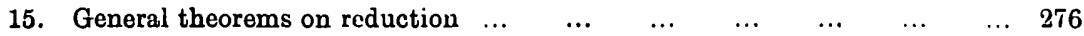

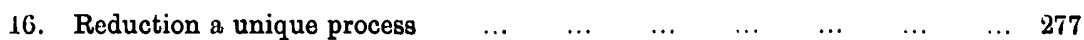

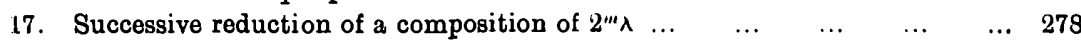

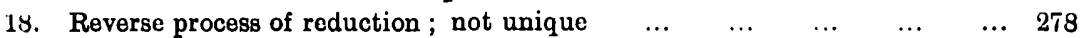

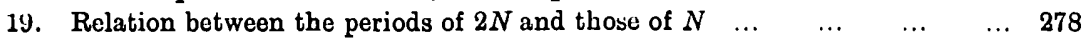

20. Reduction of a regular or an even alien composition; and converse theorcm 279 


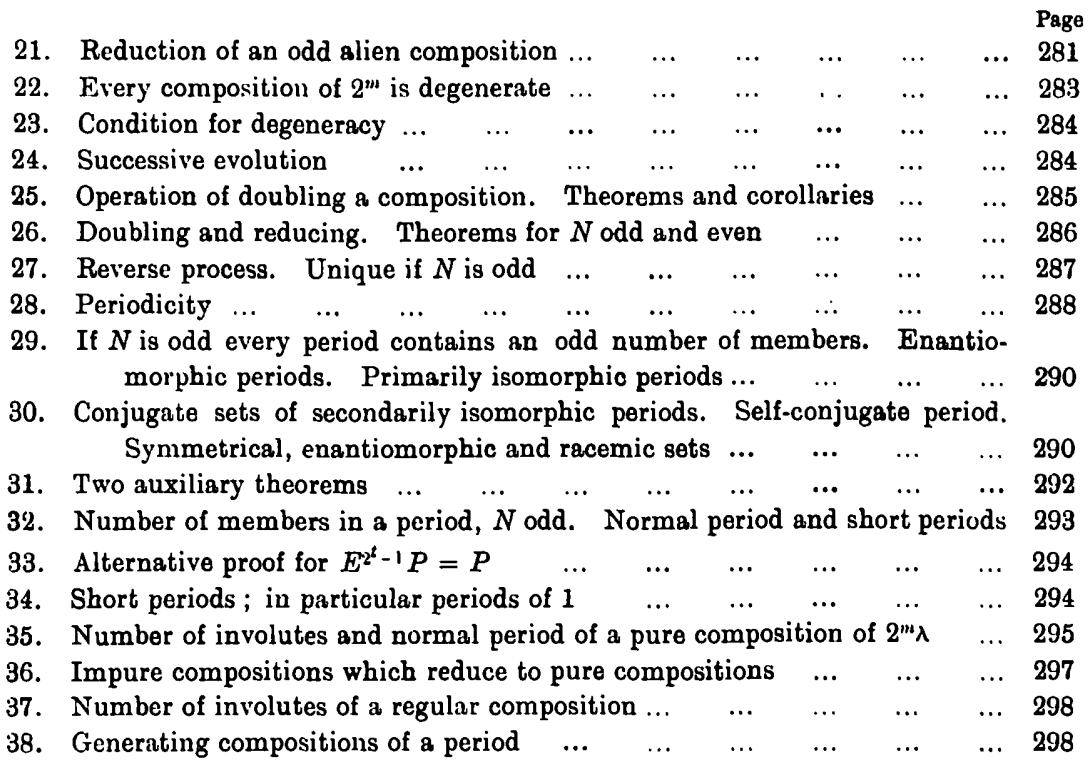

II.

Enumeration of Cyclic Compositions.

39. Number of compositions and of self-inverse compositions. Notation for $\begin{array}{llllllll}\text { number of cyclic compositions } & \ldots & \ldots & \ldots & \ldots & \ldots & \ldots & 301\end{array}$

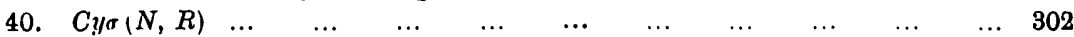

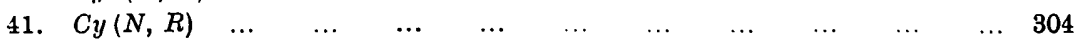

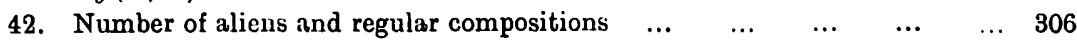

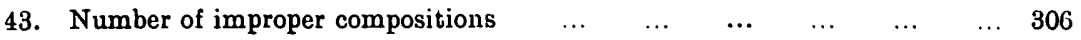

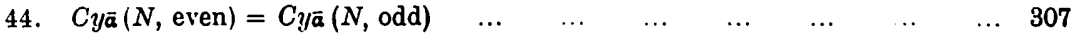

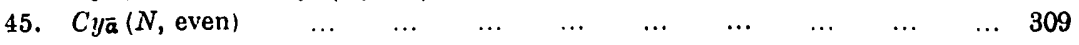

Appendix.

I. Table of numbers of cyclic compositions and periods up to $N=20$.

II. Generating compositions of the periods, and sets of periods from $N=3$ to $N=15,17,19$.

1. Consider a collection of $s$ symbols and let a row of $N$ of these be arranged in any way with or without repetitions, and imagine this row to be repeated indefinitely in both directions, or, what comes to the same thing, to be arranged in a circle. We shall call this a row of order $N$. Now let this row be endowed with the capacity of generating another row by the following process. Let the symbol $\alpha$ followed by the symbol $\beta$ be represented by the symbol $\lambda$, one of the set. It is convenient to imagine the symbols as operations which are combined according to a given multiplication table, so that the sequence $\alpha \beta$ is represented by the product 
$\alpha \beta=\lambda$. Then, the multiplication table being given, any row $S$ will generate uniquely another row $T$ of order $N$ in the $s$ symbols. Thus, if $\alpha \beta=\lambda, \beta \gamma=\mu, \gamma \delta=\nu, \ldots, \alpha \beta \gamma \delta \ldots$ generates $\lambda \mu \nu \ldots$. We shall call $T$ the first evolute of $S$. This row, again, will generate another row of order $N$. Then, since the number of arrangements, with or without repetitions, of $N$ things out of $s$ things is finite, when this process is continued we must, after a finite number of operations, return to one of the previous rows. From this row onwards the rows form a period. Conversely, if the row $S$ produces the row $T$, we may reverse the process and obtain $S$ from $T$, but in general there are several rows which will all produce $T$ and the reverse process is not unique. If $T$ is the first evolute of $S$, we shall call $S$ a first involute of $T$.

The multiplication table of the symbols may be fixed quite arbitrarily. As there are $s^{2}$ pairs, the order being taken into account, there may be $s$ or more pairs corresponding to a single symbol. If the symbol $\alpha$ corresponds to no pair, any row containing $\alpha$ will, of course, possess no involute.

2. An interesting special case is that in which for every symbol $a$, the pairs $\alpha a, \alpha \beta, \alpha \gamma, \ldots$ are all represented by different symbols, and for every symbol $\alpha$ the pairs $\alpha \alpha, \beta \alpha, \gamma \alpha, \ldots$ are all represented by different symbols. In this case, in general, every row has $s$ involutes. For, consider the row $\lambda_{1} \lambda_{2} \ldots \lambda_{N}$. $\lambda_{1}$ represents $s$ different pairs. Suppose one of them to be $\alpha_{1} \alpha_{2}$. Then there is just one symbol $\alpha_{9}$, such that $\alpha_{2} \alpha_{3}$ is represented by $\lambda_{2}$, and the row can only be continued in one way. It may happen that some of these $s$ involutes are the same.

If $\alpha_{N} \alpha_{1}$ is represented by $\lambda_{1}$ the involute is of order $N$. But if $\alpha_{N} \alpha_{1}$ is not represented by $\lambda_{1}$, we have $a_{N} \alpha_{1}^{\prime}$ represented by $\lambda_{1}$, then $\alpha_{N}^{\prime} \alpha_{1}^{\prime \prime}$, and so on, until we get finally $\alpha_{N}^{(t-1)} \alpha_{1}$ represented by $\lambda_{1}$. Then the involute is of order $t N$, and this multiple involute counts as $t$ of the possible $s$ involutes.

A still more special case is that in which the symbols can be represented by the operations of a group, or, let us say, form a group, and in particular an Abelian group.

3. To investigate the order of the involutes of a row in the case where the symbols form an Abelian group, consider the row

$$
\lambda_{1} \lambda_{2} \ldots \lambda_{N},
$$


and let

$$
\begin{gathered}
\alpha_{1} \alpha_{2}=a_{1}^{\prime} \alpha_{2}^{\prime}=\lambda_{1}, \\
\alpha_{2} \alpha_{3}=a_{2}^{\prime} \alpha_{3}^{\prime}=\lambda_{2}, \\
\cdots \quad \cdots \quad \cdots \quad \cdots \\
\alpha_{N-1} \alpha_{N}=\alpha_{N-1}^{\prime} \alpha_{N}^{\prime}=\lambda_{N-1} .
\end{gathered}
$$

Then

$$
\alpha_{2}=a_{1}^{-1} a_{1}^{\prime} a_{2}^{\prime} \text {, }
$$

therefore

$$
a_{2} \alpha_{9}=a_{1}^{-1} a_{1}^{\prime} a_{2}^{\prime} a_{3}=a_{2}^{\prime} a_{3}^{\prime}, \quad \text { i.e., } a_{1}^{\prime} a_{3}=a_{1} a_{3}^{\prime} ;
$$

also

$$
a_{3}=a_{1}^{\prime-1} a_{1} \alpha_{3}^{\prime} \text {, }
$$

therefore $\quad a_{3} \alpha_{4}=\alpha_{1}^{\prime-1} a_{1} \alpha_{3}^{\prime} \alpha_{4}=a_{3}^{\prime} \alpha_{4}^{\prime}, \quad$ i.e., $a_{1} \alpha_{4}=a_{1}^{\prime} \alpha_{4}^{\prime}$;

and generally

$$
\begin{aligned}
a_{1}^{\prime} \alpha_{2 r-1} & =a_{1} a_{2 r-1}^{\prime}, \\
a_{1} \alpha_{2 r} & =a_{1}^{\prime} a_{2 r}^{\prime} .
\end{aligned}
$$

Hence, if $N$ is odd and $=2 m+1$,

$$
\begin{gathered}
a_{2 m+1} \alpha_{1}^{\prime}=a_{2 m+1}^{\prime} \alpha_{1}=\lambda_{N}, \\
\lambda_{1} \lambda_{2} \ldots \lambda_{2 m+1} \\
a_{1} a_{2} \ldots \alpha_{2 m+1} a_{1}^{\prime} a_{2}^{\prime} \ldots \alpha_{2 m+1}^{\prime},
\end{gathered}
$$

und the involute of is

and is thus of order $2 N$, unless $a_{1}^{\prime}=a_{1}$, when it is of order $N$.

Again, if $N$ is even and $=2 m$,

$$
\alpha_{2 n} \alpha_{1}^{\prime}=a_{1}^{-1} a_{1}^{\prime} a_{2 m}^{\prime} a_{1}^{\prime}=a_{2 m}^{\prime} a_{1}^{\prime 2} a_{1}^{-1} \text {. }
$$

Let

$$
a_{1}^{\prime 2} \alpha_{1}^{-1}=a_{1}^{\prime \prime},
$$

then

$$
a_{2 m}^{\prime} \alpha_{1}^{\prime \prime}=a_{1}^{\prime-1} \alpha_{1}^{\prime \prime} a_{2 m}^{\prime \prime} a_{1}^{\prime \prime}=a_{2 m}^{\prime \prime} \alpha_{1}^{\prime-1} \alpha_{1}^{\prime 4} \alpha_{1}^{-2}=a_{2 m}^{\prime \prime} a_{1}^{\prime 3} \alpha_{1}^{-2} \text {. }
$$

Let

$$
\alpha_{1}^{\prime 3} \alpha_{1}^{-2}=a_{1}^{\prime \prime},
$$

then, similarly,

$$
a_{2 m}^{\prime \prime} a_{1}^{\prime \prime \prime}=a_{2 m}^{\prime \prime \prime} a_{1}^{\mathrm{iv}} \text {, }
$$

where

$$
\alpha_{1}^{\prime 4} \alpha_{1}^{-3}=\alpha_{1}^{\text {iv }} \text {. }
$$

Suppose

$$
\alpha_{1}^{(p)}=a_{1}^{(q)},
$$

where

$$
p>q \text {. }
$$

Then

$$
\alpha_{1}^{\prime p} \alpha_{1}^{-\rho+1}=a_{1}^{\prime \prime} \alpha_{1}^{-q+1}, \quad \text { i.e., } \quad \alpha_{1}^{p-q}=\alpha_{1}^{p-q} .
$$

Then

$$
\alpha_{1}^{(p-q)}=\alpha_{1}^{p-q} \alpha_{1}^{-p+q+1}=\alpha_{1}^{p-q} \alpha_{1}^{-p+q+1}=\alpha_{1},
$$

and if $t$ is the smallest index for which $\alpha_{1}^{\prime t}=a_{1}$, the involute of $\lambda_{1} \ldots \lambda_{2 m}$ will be of order $2 \mathrm{tm}$, and $t$ must be a factor of the order of the group, i.e., of $s$. Hence, if $s$ is prime, the involute of any row of order $N$ will be a row of order either $N, 2 N$ or $s N$. Also for a row of even order, if an 


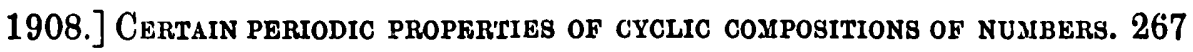

involute of $S$ is $R_{1} R_{2} \ldots R_{t}$, then the evolutes of $R_{1}, R_{2}, \ldots$ are all the same, though not equal to $S$, for $a_{2 m} \alpha_{1}=a_{2 i l}^{\prime} \alpha_{1}^{\prime}=\ldots$.

To find the condition that the involute of a row of order $N$ may also be a row of order $N$.

We have

$$
\begin{gathered}
\alpha_{1} \alpha_{2}=\lambda_{1}, \quad \alpha_{2} \alpha_{8}=\lambda_{2}, \ldots, \quad a_{N-1} \alpha_{N}=\lambda_{N-1}, \quad \alpha_{N} a_{1}=\lambda_{N} . \\
\alpha_{2}=\alpha_{1}^{-1} \lambda_{1}, \text { therefore } a_{2} \alpha_{3}=\alpha_{1}^{-1} \lambda_{1} \alpha_{8}=\lambda_{2} ;
\end{gathered}
$$

hence

$$
\lambda_{1} \alpha_{9}=\lambda_{2} \alpha_{1} \text {. }
$$

$$
a_{3}=\lambda_{1}^{-1} \lambda_{2} \alpha_{1} \text {, therefore } a_{8} \alpha_{4}=\lambda_{1}^{-1} \lambda_{2} \alpha_{1} \alpha_{4}=\lambda_{9} \text {; }
$$

hence

$$
\lambda_{2} a_{1} a_{4}=\lambda_{1} \lambda_{3} \text {. }
$$

Similarly,

$$
\begin{aligned}
\lambda_{1} \lambda_{3} \ldots \lambda_{2 r-1} \alpha_{2 r+1} & =\lambda_{2} \lambda_{4} \ldots \lambda_{2 r} \alpha_{1}, \\
\lambda_{1} \lambda_{3} \ldots \lambda_{2 r-1} & =\lambda_{2} \lambda_{4} \ldots \lambda_{: r-2} \alpha_{1} \alpha_{2 r} .
\end{aligned}
$$

Let $N=2 m+1$.

$$
\begin{aligned}
a_{2 m+1} \alpha_{1}= & \lambda_{2 m+1}=\lambda_{2} \lambda_{1} \ldots \lambda_{2 m} \alpha_{1}\left(\lambda_{1} \lambda_{3} \ldots \lambda_{2 m-1}\right)^{-1} a_{1}, \\
\text { i.e., } \quad & \lambda_{1} \lambda_{3} \ldots \lambda_{2 m+1}=\lambda_{2} \lambda_{4} \ldots \lambda_{2 m} a_{1}^{2} .
\end{aligned}
$$

Let $N=2 m$.

$$
\begin{aligned}
\alpha_{2 m} \alpha_{1}= & \lambda_{2 m}=\lambda_{1} \lambda_{3} \ldots \lambda_{2 m-1}\left(\lambda_{2} \lambda_{4} \ldots \lambda_{2 m-2}\right)^{-1}, \\
\text { i.e., } & \lambda_{1} \lambda_{3} \ldots \lambda_{2 m-1}=\lambda_{2} \lambda_{4} \ldots \lambda_{2 m} .
\end{aligned}
$$

Hence (1) A row of order $2 m+1$ has involutes of order $2 m+1$ in which the first symbol $\alpha_{1}$ is determined by

$$
\alpha_{1}^{2}=\left(\lambda_{1} \lambda_{3} \ldots \lambda_{2 m+1}\right)\left(\lambda_{2} \lambda_{4} \ldots \lambda_{2 m}\right)^{-1},
$$

and every other involute, starting with a symbol $\alpha_{1}$ which does not satisfy this equation is of order $2(2 m+1)$.

(2) A row of order $2 m$ has all its involutes of order $2 m$ if

$$
\lambda_{1} \lambda_{3} \ldots \lambda_{2 m-1}=\lambda_{2} \lambda_{4} \ldots \lambda_{: m n} ;
$$

but if this condition is not satisfied its involutes will be of order $2 \mathrm{mt}$, where $t$ is the smallest index for which $\alpha_{1}^{\prime t}=\alpha_{1}^{t}$, and $t$ is a factor of $s$.

Two particular cases may be noticed.

(1) $s$ an odd prime.

A row of order $2 m+1$ has one involute of order $2 m+1$ and $\underline{\underline{1}}(s-1)$ involutes of order $2(2 m+1)$. 
A row of order $2 m$ has either $s$ involutes of order $2 m$ or one involute of order $2 m s$.

(2) $s$ a power of 2 , and the group of type $(1,1,1, \ldots)$.

A row of order $N$ has either $s$ involutes of order $N$ or $\frac{1}{2} s$ involutes of order $2 N$ according as $\lambda_{1} \lambda_{3} \ldots$ is, or is not, equal to $\lambda_{2} \lambda_{4} \ldots$.

I.

4. The simplest case, and that to which our attention will be confined, is the case of two symbols which we may denote by + and - , and connect by the usual law of signs

$$
++=--=+,+-+=-,
$$

i.e., a permanence of sign is denoted by + and a change of sign by - . These symbols may evidently be represented by the operations of an Abelian group of order 2 .

In order to represent a row of order $N$ let $(p)$ represent a sequence of $p$ plus signs and $q$ a sequence of $q$ minus signs. Then the row may be represented by

where

$$
\left(p_{1}\right) q_{1}\left(p_{2}\right) q_{2} \ldots\left(p_{r}\right) q_{r}
$$

$$
\sum_{1}^{r}\left(p_{\rho}+q_{\rho}\right)=N \text {. }
$$

This represents a partition of $N$ into $2 r$ parts. Every row of order $N$ is therefore represented by a partition of $N$ into an even number of parts, where the cyclic order of the parts is taken into account, and the "plus" and "minus" parts, which follow alternately, are distinguished.

A partition of $N$ in which the order of the parts is essential has been called by Glaisher* a composition of $N$. A partition in which only the cyclic order of the parts is essential will here be called a cyclic composition. If there is no risk of confusion we may use simply the term composition. We may say then that every row of order $N$ is represented by an even cyclic composition. We shall have also to consider compositions of $N$ with an odd number of parts, or odd compositions. From the present point of view an odd cyclic composition standing by itself condenses at once into an even cyclic composition, as $(p) q(r)$ into $(p+r) q$, but this process is to be excluded and an odd cyclic composition is to be considered

* Cf. MacMahon, "The Theory of Perfect Partitions of Numbers and the Compositions of Multipartite Numbers," Messenger of Mathematics, Vol. xx. (1891), pp. 103-119. 
1908.] Certain periodic properties of cyclic compositions of numbers. 269

as imperfect and as giving a row only when conjoined with the alternate composition, as $(p) q(r) p(q) r$.

Let us attempt to extend this nomenclature to the general case of $s$ symbols. The number which expresses a collection of $s$ different kinds of objects, $n_{1}$ of $a_{1}, n_{2}$ of $a_{2}, \ldots$ is called a multipartite number, $\uparrow$ and is written

$$
\left(n_{1} n_{2} \ldots\right) \text {. }
$$

A partition of such multipartite number is

$$
\left(a_{1} a_{2} \ldots, b_{1} b_{2} \ldots, \ldots\right) \text {, }
$$

where $\Sigma a_{1}=n_{1}, \Sigma a_{2}=n_{2}, \ldots$, and if the order of the parts $a_{1} a_{2} \ldots$, $b_{1} b_{2} \ldots, \ldots$ is essential, we have a composition of the $s$-partite number. In the present theory not only the cyclic order of the parts is essential, but also the cyclic order of the whole row. Two compositions, as

$$
\alpha a \beta \beta \gamma \beta \alpha \beta \gamma \text { and } \beta \gamma \beta \alpha \beta \gamma \alpha \alpha \beta \text {, }
$$

represented by $221,010,111$ and $011,010,111,210$,

are in this theory considered identical; so that a row can be represented in many ways by a composition of the same s-partite number. Further, the evolute of a row which is represented by a composition of an $s$-partite number is not in general represented by a composition of the same $s$-partite number. Thus, if the multiplication table for the symbols $a, \beta, \gamma$ is

\begin{tabular}{l|lll}
\multicolumn{1}{c}{} & \multicolumn{1}{c}{$\alpha$} & $\beta$ & $\gamma$ \\
\cline { 2 - 4 }$\alpha$ & $a$ & $\beta$ & $\gamma$ \\
$\beta$ & $\beta$ & $\gamma$ & $u$ \\
$\gamma$ & $\gamma$ & $\alpha$ & $\beta$
\end{tabular}

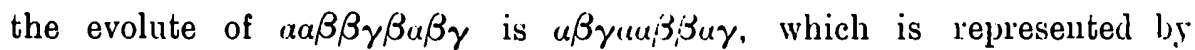
$111,220,101$, a composition of the tripartite number 432 , while $\iota \alpha_{1} \beta \beta \gamma \beta a \beta \gamma$ is represented by a composition of 342 . In the particuliar ciise of two symbols, again, a row would be represented by a composition of a bipartite number denoting the number of plus and minus signs; but here again, for example, the evolute of 23 is 1121 , a composition of 32 , not 23 . The multipartite number is therefore not invariable during the operations, though the total number of symbols is, and we gain nothing by representing a row by a composition of a multipartite number. In the case of two symbols we shall find that for most purposes the distinction between

* See below, $\$ 5$.

† MacMahon, Messenger of Mathematics, Vol. xx., p. 107. Also "Memoir on Symmetric Functions of the Roots of Systems of Equations," Phil. Trans., Vol. CLxxxi., A. (1\$90). pp. 4S1-536. 
the plus and minus parts can be disregarded, and a row can be represented by an even cyclic composition of the unipartite number $N$.

\section{Rules for Evolution.}

Consider the row $(p) q(r) s . \quad(p)$ denotes a sequence of $p$ plus signs, and on evolution gives $p-1$ plus signs, similarly $q$ gives $q-1$ plus signs. Also in passing from $(p)$ to $q$ or from $q$ to $(r)$ we have one change of sign, which is represented by a minus. Hence the first evolute of (p) $q(r) s$ is

$$
(p-1), 1,(q-1), 1,(r-1), 1,(s-1), 1 .
$$

If $q=1$, this rule fails. We have then two consecutive changes of sign from $(p)$ to $(r)$, and this is represented by 2 . Similarly a sequence of $q$ oues gives $q+1$ minus signs. Hence, denoting a sequence of $q$ ones by $1^{\prime \prime}$, the first evolute of

is

$$
\text { (p), } 1^{i},(r), \ldots
$$

$$
(p-1), q+1,(r-1), \ldots .
$$

We shall denote the $r$-th evolute of $P$ by $E^{r} P$ and an $r$-th involute by $E^{-r} P$, remembering, however, that the inverse operation is not unique.

The first evolutes of $(p) q(r) s \ldots$ and $p(q) r(s) \ldots$ are the same. Two rows which only differ by interchanging the plus and minus signs are called alternate. The alternate of $P$ will be denoted by $\bar{P}$.

\section{Rule for Involution.}

A row $(p) q(r) s \ldots$ of order $N$ has, in general, two involutes

$$
(p+1), 1,(1), 1, \ldots \text { and } p+1,(1), 1,(1), \ldots,
$$

and these are alternate rows, $P, \bar{P}$, of order $N$. But the involute may be of order $2 N$. In this case $P, \bar{P}$ are imperfect and the single involute is represented by $P \bar{P}$.

7. A row which has two involutes of the same order as itself will be called proper, one which has one involute of double the order improper.

A row of $2 N$, such as $P \bar{P}$, in which $P$ is imperfect, will be called regular. Its first evolute is $Q Q$, where $Q$ is an even composition, but this is just a row of $N$. Considered as a row of $2 N, Q Q$ will be called alien.

A more general definition of an alien may be given.

An alien of order $k N$ consists of a non-alien row or composition of $N$ 
repeated $k$ times, and it is said to appertain to $N$; it will be called an even or an odd alien according as $k$ is even or odd. The following case is excluded. twice.

A regular row of $2 N$ consists of a non-alien composition of $N$ repeated

A row, one of whose successive evolutes is alien is said to be degenerate. A row of $N$ whose successive evolutes are all non-alien rows of $N$ is said to be irreducible.

If $N$ is odd there can be no regular rows, and therefore no degenerate rows, but there may be aliens. If $N$ is prime every row is irreducible.

The evolutes of any row, if they are not alien, are proper.

The involutes of any improper row are degenerate.

8. If an irreducible row of $N$ be repeatedly evolved, we shall return after a finite number of operations to a previous evolute. Suppose

$$
E^{n} P=E^{r} P .
$$

Then we have a period of $n-r$, and every irreducible row either belongs to a period or evolves to a row belonging to a period. A row belonging to a period is called a pure row. Every pure row is proper.

Let the rows of a period be involved successively. Each row gives first the previous row of the period and its alternate. Each of the alternates, if they are proper, then gives two other rows, and each of these again two others, until we arrive at improper rows. This must happen at some stage since the number of compositions is finite, and since no further periods can arise, and of these improper rows there is, of course, a finite number. The set of all these rows, any one of which evolves finally to a pure row of the same period, forms the complete set of rows belonging to the period. If these are arranged in a scheme such that each row is the evolute of the next row to the left and an involute of the next to the right, we have on the right the period and on the left a dichotomous arborescence ending with improper rows.

If a row is degenerate it is either a regular row or an involute of a regular row. This is obvious from the way in which a regular row arises.

\section{Condition that a row may be proper.}

The first involute of

$$
\left(p_{1}\right) q_{1}\left(p_{2}\right) q_{2} \ldots\left(p_{a}\right) q_{a},
$$

$$
p_{1}+1,1^{q_{1}-1}, p_{2}+1,1^{1_{2}-1}, \ldots \text {, }
$$


and the number of parts must be even. But the number of parts is

$$
a+\Sigma(q-1)=\Sigma q
$$

Hence the condition is that the sum of the "minus" parts, or the number of minus signs, must be even.

This can be deduced also from the condition in the general case,

$$
\lambda_{1} \lambda_{3} \ldots=\lambda_{2} \lambda_{4} \ldots
$$

This gives $\quad \lambda_{1} \lambda_{2} \lambda_{3} \ldots=\left(\lambda_{1} \lambda_{3} \ldots\right)^{2}=+$,

which proves the result.

If $N$ is even, two alternates are either both proper or both improper. If $N$ is odd, of two alternates one is proper and the other improper.

10. A row which reads the same way backwards as forwards is called symmetrical.* Unsymmetrical rows occur as pairs of enantiomorphs.

The evolutes of a symmetrical row are symmetrical, and the successive corresponding evolutes of two enantiomorphs are either enantiomorphs or identical and symmetrical.

Also, if one member of a period is symmetrical, all the members of the period are symmetrical. Such a period will be called a symmetrical neriod.

Again, if one member of a period be unsymmetrical, no member of the complete set is symmetrical.

If + two enantiomorphs belong to the same period every row is accompanied in the period by its enantiomorph.

For, if $P$ and $P^{\prime}$ both occur and the successive evolutes of $P$ are $Q R S \ldots$, the successive evolutes of $P^{\prime}$ are $Q^{\prime} R^{\prime} S^{\prime} \ldots$ The period must, therefore, be of the form $P Q R \ldots X P^{\prime} Q^{\prime} R^{\prime} \ldots X^{\prime}$. If this does not happen then we have pairs of enantiomorphic periods and sets.

- MacMuhon [Phil. Traus., Vol. clxxxiv., A. (1893), p. 838] calls a composition which is formed by reversing the order of the parts of another composition, the inverse of the other composition, and a composition which reads the same way backwards as forwards a selfinverse composition. These terms will be retained when we are dealing with ordinary compositions, but as it will be convenient to speak of a cyclic composition as being symmetrical about some part or pair of parts the term symmetrical seems to be more useful for our purpose than either self-inverse or reversible.

$\dagger$ It is proved later $(\S 29)$ for the case wherc $N$ is odd that this is impossible. 
11. It will be convenient now to drop the explicit distinction between a row and its alternate. A row will then be represented simply by a cyclic composition of the unipartite number $N$. We may say then that every composition, unless it is regular, has two first involutes, viz., the involutes of $P$ will be $E^{-1} P$ and $E^{-1} \vec{P}$. These are not, of course, alternates, and they are identical if $P$ is regular, for then $\bar{P}=P$. Then a pure composition has two involutes, one of which belongs to the period. If $N$ is odd, one involute is of order $2 N$, and a composition is either alien or pure. In this case the complete set contains only the period. If $N$ is even the distinction between proper and improper compositions survives.

12. Two cyclic compositions which have the same first evolute are conjugate in the corresponding sense to that in which the term is used by MacMahon for ordinary compositions.

Adapting his graphical representation we may represent a cyclic composition by a series of nodes and links in a circle. The number of links between two successive nodes denotes a part of the composition. The conjugate is obtained by suppressing the existing nodes and putting nodee at the other angular points. Thus we have a cyclic composition of 12 and its conjugate.
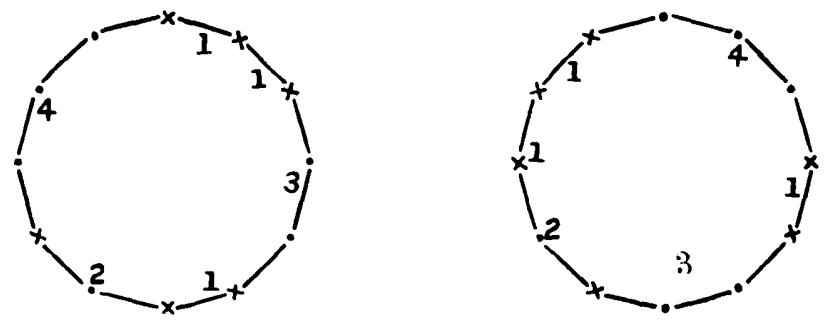

The cyclic composition represented by

$$
a_{1}, 1^{a_{1}}, a_{2}, 1^{a_{2}}, \ldots,
$$

will have for its conjugate

$$
1^{a_{1}-2}, a_{1}+2,1^{a_{2}-2}, a_{2}+2, \ldots,
$$

and the first evolute of either is

$$
a_{1}-1, a_{1}+1, a_{2}-1, a_{2}+1, \ldots .
$$

Graphically, the first evolute is obtained by replacing links by points and points by links, and putting nodes at points which replace links un- 
symmetrically bounded. Thus the first evolute of the above compositions is represented by

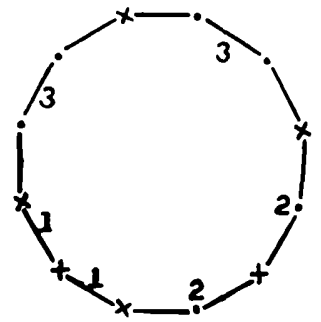

If $R$ is the number of parts in a cyclic composition, the number of parts in the conjugate is $N-R$. Hence, if $N$ is odd the conjugate cyclic composition is imperfect.

Conjugate compositions are always distinct, but a cyclic composition may be self-conjugate. This requires that $N$ be even and $R=\frac{1}{2} N$, and the composition is the first involute of a regular composition or of an alien consisting of a regular composition repeated. Thus $1^{2} 3412$ is selfconjugate and its first evolute is $321321 ; 1^{2} 41^{2} 4$ is self-conjugate and its first evolute is 3333. A self-conjugate cyclic composition is therefore always degenerate; but the converse is not true, thus 12321223 is degenerate ( $\$ 22)$, but its conjugate is 32123221 .

A cyclic composition may be inversely conjugate, i.e., its conjugate may be the original composition read backwards. In this case also $R=\frac{1}{2} N$, and the first evolute must be symmetrical. E.g., $1^{2} 2242$ is inversely conjugate, its conjugate being $4221^{2} 2$, and its tirst evolute $31^{4} 31^{2}$.

13. We shall proceed now to consider some other processes to which the compositions can be subjected.

Reduction of a cyclic composition of $2 N$, modulo $N$.

Take any cyclic composition $P$ of $2 N$ in which one of its parts exceeds $N$. Then, on subtracting $N$ from this part, we get a cyclic composition of $N$ which will be denoted by $P(\bmod N)$.

Consider the composition $P=p, q, N+r, s$, where

$$
p+q+r+s=N \text { and } r>1 \text {. }
$$

Evolving, we get $p-1,1, q-1,1, N+r-1,1, s-1,1$.

On reducing the original composition, modulo $N$, we get $p, q, r, s$, and its first evolute is $p-1,1, q-1,1, r-1,1, s-1,1$, and this is the same as the 
first evolute of $P$ reduced modulo $N$, i.e.,

$$
E\{P(\bmod N\}=\{E(P)\}(\bmod N) .
$$

To investigate the rules for reduction, modulo $N$, when no part exceeds $N$, we shall fix the condition represented by this equation.

If

$$
P=p, 1^{4-1}, N+1,1^{s-1}, \quad P(\bmod N)=p, 1^{4+s-1},
$$

then

$$
\{E(P)\}(\bmod N)=p-1, q, 0, s
$$

and

$$
E\left\{P(\bmod N)_{i}^{\prime}=p-1, q+s\right. \text {. }
$$

Hence

$$
q, 0, s=q+s \text {. }
$$

Next let

$$
P=p \cdot 1^{i-1}, N, 1^{s-1} \text {. }
$$

$$
\begin{gathered}
P(\bmod N)=p, 1^{q-1}, 0,1^{s-1}=p, 1^{q-2}, 2,1^{s-2} . \\
E\{P(\bmod N) !=p-1, q-1,1, s-1, \\
: E(P) !(\bmod N)=p-1, q,-1, s .
\end{gathered}
$$

Hence

$$
q,-1, s=q-1,1, s-1 \text {. }
$$

Proceeding in this way, it may be shown generally that

$$
q,-r, s=q-r, r, s-r .
$$

We may thus introduce zero and negative parts subject to the following rules:-

$$
\begin{aligned}
\text { I. } \quad p, 0, q & =p+q, \\
\text { II. } p, 0,0, q & =p, q, \\
\text { III. } p,-q, r & =p-q, q, r-q .
\end{aligned}
$$

These rules for the elimination of zero and negative parts may be taken to be quite general. It will sometimes be convenient in evolving a composition to retain zero or negative parts. A negative part will sometimes be written with the minus over the part, as $\bar{p}$.

14. Having found an interpretation for zero and negative parts, let us investigate the meaning of a negative number of ones.

A first involute of $p, q$ is $p+1,1^{t-1}$. Let us assume that this holds whether $p$ and $q$ be positive or negative, then we have

$$
E^{-1}(p, \bar{\eta})=i^{\prime}+1,1^{-(1+1)} \text {. }
$$

Also

$$
p, \bar{q}, r, s=p-q, q, r-q, s
$$

and

$$
\begin{aligned}
E^{-1}(p, \bar{q}, r, s) & =p+1,1^{-(1+1)}, r+1,1^{s-1} \\
& =p-q+1,1^{t-1}, r-q+1,1^{s-1} .
\end{aligned}
$$

Hence

$$
p, 1^{-(1+1)}, r=p-q, 1^{q-1}, r-q \text {. }
$$


If $q=0$,

and

Hence

$$
1,0, r, s=p+r, s
$$$$
E^{-1}(p+r, s)=p+r+1,1^{s-1} \text {. }
$$

$$
p, 1^{-1}, r=p+r-1 \text {, }
$$

15. General Theorems on Reduction.

I. $p_{1}-N, p_{2}, p_{9}, \ldots, p_{k}$

$$
=p_{2}, p_{3}, p_{1}, \ldots, p_{r}, N-\sum_{1}^{r} p_{\rho}, \sum_{1}^{r+1} p_{\rho}-N, p_{r+2}, \ldots, p_{k-1}, p_{k}+p_{1}-N .
$$

Assume this to be true for $r$, then we get as the next step ( $\$ 13$, III.)

$$
p_{2}, p_{3}, \ldots, p_{r}, p_{i-1}, N-\sum_{1}^{r+1} p_{\rho}, \sum_{1}^{r+2} p_{\rho}-N, p_{r+3}, \ldots, p_{k-1}, p_{k}+p_{1}-N \text {. }
$$

But the first step is

$$
N-p_{1}, p_{1}+p_{2}-N, p_{3}, \ldots, p_{k-1}, p_{k}+p_{1}-N .
$$

Hence the process is established.

II. $p-N, 1^{y}, r, 1^{s}=1^{i-(p+\lambda)}, \lambda+1,1^{p+q-\lambda^{\prime-1}}, r, 1^{s-\lambda}, p+\lambda-N$.

By Theorem I. the left-hand side becomes

$$
1^{\lambda}, N-(p+\lambda), p+\lambda+1-N, 1^{4-\lambda-1}, r, 1^{s-1}, p+1-N .
$$

Put $\lambda=N-(p+1)$, and we get

$$
\begin{aligned}
& 1^{N-(p+1)}, 1,0,1^{p+q-N}, r, 1^{s-1}, p+1-N \\
= & 1^{N-(p+1)}, 2,1^{p+l-N-1}, r, 1^{s-1}, p+1-N .
\end{aligned}
$$

By the same process this gives

$1^{\lambda}, N-(p+1-\lambda), p+\lambda+2-N, 1^{N-(1+\lambda+2)}, 2,1^{p+1-N-1}, r, 1^{s-2}, p+2-N$, and, putting

$$
\lambda=N-(p+2) \text {, }
$$

$$
\begin{aligned}
& 1^{N-(p+2)}, 1,0,2,1^{p+y-N-1}, r, 1^{s-2}, p+2-N \\
= & 1^{N-(p+2)}, 3,1^{p+q-N-1}, r, 1^{s-2}, p+2-N .
\end{aligned}
$$

Now assume the theorem for $\lambda$ such steps, and we have

$$
p+\lambda-N, 1^{N-(p+\lambda)}, \lambda+1,1^{p+\imath-\lambda^{-1}}, r, 1^{s-\lambda},
$$

which becomes at the next stage

$1^{\mu}, N-(p+\lambda+\mu), p+\lambda+\mu+1-N, 1^{N-(p+\lambda+\mu+i)}$,

$$
\lambda+1,1^{1+1+-N-1}, r, 1^{s-\lambda-1}, p+\lambda+1-N,
$$


1908.] Certain periodic properties of CyCLic compositions of numbers. 277

and, putting $\mu=N-(p+\lambda+1)$, we get

$$
\begin{aligned}
& 1^{N-(p+\lambda+1)}, 1,0, \lambda+1,1^{p+q-N-1}, \ldots \\
= & 1^{N-(p+\lambda+1)}, \lambda+2,1^{p+q-N-1}, r, 1^{s-(\lambda+1)}, p+\lambda+1-N .
\end{aligned}
$$

Hence the theorem is established.

III. $p-N, 1^{4}, r, s, 1^{t}=q+2,1^{N-(p+q+1)}, p+q+r-N, s, 1^{t+p-x-1}$.

By Theorem II. the left-hand side is equal to

$$
1^{N-(p+\lambda)}, \lambda+1,1^{p+q-N-1}, r, s, 1^{t-\lambda}, p+\lambda-N .
$$

Put $\lambda=N-p$, and we get

$$
\begin{aligned}
& N-p+1,1^{p+q-N-1}, r, s, 1^{t+p-N}, 0 \\
= & N-p+2,1^{p+q-N-1}, r, s, 1^{t+p-N-1} \\
= & q+2,1^{N-1-p-q}, p+q+r-N, s, 1^{t+p-N-1}
\end{aligned}
$$

Hence the theorem is true.

We may, of course, interpolate any parts between the $r$ and the $1^{t}$, as these are not affected by the process. Also, in all three theorems $N$ may have any value.

16. We have now to show that the process of reduction is unique.

Let

where

$$
P=p_{1}, p_{2}, \ldots, p_{k},
$$

$$
\Sigma p=2 N \text {. }
$$

By Theorem I.,

$$
\begin{aligned}
& p_{1}, p_{2}-N, p_{3}, \ldots, p_{k} \\
= & p_{1}+p_{2}-N, p_{8}, \ldots, p_{r}, N-\sum_{2}^{r} p_{\rho}, \sum_{2}^{r+1} p_{\rho}-N, p_{r+2}, \ldots, p_{k} .
\end{aligned}
$$

Putting $r=k-1$, we get

$$
\begin{aligned}
& p_{1}+p_{2}-N, p_{8}, \ldots, p_{k-1}, N-\sum_{y=}^{k-1} p_{\rho}, \sum_{2=}^{k} p_{\rho}-N \\
= & p_{1}+p_{2}-N, p_{3}, \ldots, p_{k-1}, p_{1}+p_{k}-N, N-p_{1} \\
= & p_{2}, p_{8}, \ldots, p_{k-1}, p_{k}, p_{1}-N .
\end{aligned}
$$

Then, since

$$
p_{1}, p_{2}-N, p_{8}, \ldots, p_{k}=p_{1}-N, p_{2}, p_{3}, \ldots, p_{k},
$$

therefore

$p_{1}, p_{2}, p_{3}-N, p_{4}, \ldots, p_{k}=p_{1}, p_{2}-N, p_{3}, \ldots, p_{k}$

and so on.

$$
=p_{1}-N, p_{\mathbb{8}}, p_{8}, \ldots, p_{k},
$$

Hence the result is proved generally, and reduction, modulo $N$, is a unique process. This result is true whatever the number of parts may be, even or odd. 
By a repetition of this process it is always possible to arrive at a composition free from negative parts, for, on the reduction by the above rule for negative parts, the negative purts which may be introduced, as others are made positive; continually become smaller numerically. Thus from $p, q,-r, s$ we get $p, q-r, r, s-r$, and, if $q-r$ is negative, $r-q<r$. These parts will ultimately either vanish or become positive. In actual practice it is advisable naturally to subtract $N$ from the largest part.

17. Successive reduction of a composition of $2^{m} \lambda$ to a composition of $\lambda$.

Any composition of $2^{m} \lambda$ may, by the above process, be reduced uniquely to a composition of $2^{m-1} \lambda$. This, again, by a repetition of the same process, may be reduced to a composition of $2^{m-2} \lambda$, and so on until, finally, we reach a composition of $\lambda$, and the final result is unique. Also the evolute of such a composition when treated in the same way always leads to the evolute of the corresponding composition.

This reduction may be effected by separate stages in the manner just indicated, or it may be effected at one step, or the reduction to the various moduli, $2^{m-1} \lambda, 2^{m-s} \lambda, \ldots$, may be made in any order. This is made clear if we retain the zeroes which may arise; then the effect of the reduction to any modulus can be traced throughout all the stages, and it is immaterial from what part this modulus be subtracted.

18. The reverse process may also be performed, i.e., to obtain a composition of $2 N$ from a composition of $N$. Zero or negative parts may be first introduced and the composition transformed in any way according to the preceding rules. Then $N$ is added to any part and the whole composition again transformed until zero and negative parts are eliminated. The reverse process is evidently not unique. Thus 2,4 ; $1,1,1,3 ; 1,5$; and $1,1,2,2$ all reduce, modulo 3 , to 1,2 .

19. If $P$ belongs to a period of $n, P(\bmod N)$ will belong to a period of $n^{\prime}$, a factor of $n$, and, if $P(\bmod N)$ belongs to a period of $n^{\prime}, P$ will evolve to a composition belonging to a period containing a multiple of $n^{\prime}$.

We have

$$
E^{n} P=P, \quad\left(E^{n} P\right)(\bmod N)=E^{n}\{P(\bmod N)\}=P(\bmod N),
$$

and also

$$
E^{n^{\prime}}\{P(\bmod N)\}=P(\bmod N) \text {; }
$$

therefore $n$ is a multiple of $n^{\prime}$. 
1908.] Certain periodic properties of cyolic compositions of numbers. 279

20. A regular or an even alien composition of $2 N$ reduces; modulo $N$, to a single part, and, when reduced, modulo $2 N$, vanishes.

Consider the composition

$$
P=p_{1}, p_{2}, p_{8}, \ldots, p_{k}, p_{1}, p_{2}, \ldots, p_{k},
$$

where

$$
\sum_{1}^{k} p_{p}=N \text {. }
$$

We have

$$
\begin{aligned}
& p_{1}-N, p_{2}, p_{8}, \ldots, p_{k}, p_{1}, p_{2}, \ldots, p_{k} \\
= & p_{2}, p_{3}, \ldots, p_{k-1}, N-\sum_{1}^{k-1} p_{\rho}, \sum_{1}^{k} p_{\rho}-N, p_{1}, \ldots, p_{k-1}, p_{1}+p_{k}-N \\
= & p_{2}, p_{3}, \ldots, p_{k-1}, p_{k}, 0, p_{1}, p_{2}, \ldots, p_{k-1}, p_{1}+p_{k}-N \\
= & p_{1}+p_{k}-N, p_{2}, p_{3}, \ldots, p_{k-1}, p_{k}+p_{1}, p_{2}, \ldots, p_{k-1} \\
= & p_{2}, p_{3}, \ldots, p_{k-2}, p_{k-1}+p_{k}+p_{1}, p_{2}, \ldots, p_{k-2}, p_{k-1}+p_{k}+p_{1}-N .
\end{aligned}
$$

Proceeding in this way we get

$$
p_{1}+\sum_{r}^{k} p_{\rho}-N, p_{2}, p_{3}, \ldots, p_{r-1}, p_{1}+\sum_{i}^{k} p_{\rho}, p_{2}, \ldots, p_{r-1} ;
$$

and, putting $r=3$,

$$
\begin{aligned}
p_{1}+\sum_{3}^{k} p_{\rho}-N, p_{2}, p_{1}+\sum_{3}^{k} p_{\rho}, p_{2} & =-p_{2}, p_{2}, N-\rho_{2}, p_{2} \\
& =p_{2}, 0, N-p_{2}, 0 \\
& =N .
\end{aligned}
$$

Conversely, $P$ will condense to a single part when reduced, modulo $N$, only if it is regular or an even alien.

At each step of the reduction, except the last, there can be only one condensation whereby the number. of parts is reduced by 2 , and the penultimate result must be of the form $p, 0, q, 0$; hence the number of

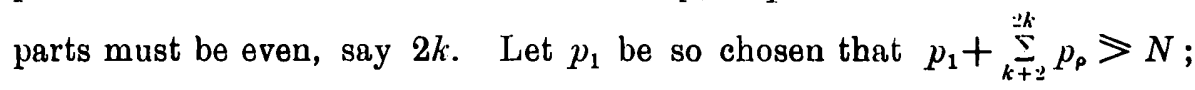
then, since the negative part at the end of the series after the $r$-th step is $p_{1}+\sum_{2 k-r+1}^{2 k} p_{p}-N$, there cannot be more than $k-1$ steps. But the number of condensations required up to the second last step is

$$
\frac{1}{2}(2 k-4)=k-2 .
$$

Hence there must be $k-1$ steps, and one condensation must occur at each step except the last, where there are two. 
We have

$$
\begin{aligned}
& p_{1}-N, p_{2}, \ldots, p_{2 k} \\
& \quad=p_{2}, p_{3}, \ldots, p_{r-1}, N-\sum_{1}^{r-1} p_{\rho}, \sum_{1}^{r} p_{\rho}-N, p_{r+1}, \ldots, p_{2 k-1}, p_{1}+p_{2 k}-N .
\end{aligned}
$$

In order that condensation may occur we must have

$$
\sum_{1}^{\iota_{1}} p_{p}=N
$$

for some value of $l_{1}$. Also $p_{1}+\sum_{k+2}^{2 k} p_{\rho}>N$,

therefore

$$
\sum_{2}^{k+1} p_{\rho} \gtrless N=p_{1}+\sum_{2}^{l_{1}} p_{\rho}
$$

therefore

$$
l_{1} \gtrless k \text {. }
$$

We have then as the first step

$$
\begin{aligned}
& p_{1}+p_{2 k}-N, p_{2}, \ldots, p_{l_{1}-1}, p_{l_{1}}, 0, p_{l_{1}+1}, p_{l_{1}+2}, \ldots, p_{2 k-1} \\
= & p_{1}+p_{2 k}-N, p_{2}, \ldots, p_{l_{1}-1}, p_{l_{1}}+p_{l_{1}+1}, p_{l_{1}+2}, \ldots, p_{2 k-1} .
\end{aligned}
$$

For the second step we have

$$
p_{2}, p_{3}, \ldots, p_{r-1}, N-\sum_{1}^{r-1} p_{\rho}-p_{2 k}, \sum_{1}^{r} p_{\rho}+p_{2 k}-N, p_{r+1}, \ldots ;
$$

and for condensation $\quad \sum_{1}^{l_{2}} p_{p}+p_{2 k}=N$;

therefore

$$
p_{2 k}=\sum_{l_{2}+1}^{l_{1}} p_{\rho} \text { and } l_{2}<l_{1} \text {. }
$$

This gives

$$
\begin{aligned}
p_{1}+p_{2 k}+p_{2 k-1}-N, p_{2}, \ldots, p_{l_{2}-1}, p_{l_{2}}+p_{l_{2}+1}, p_{l_{9}+2}, \ldots, \\
p_{l_{1-1}}, p_{l_{1}}+p_{l_{1}+1}, p_{l_{1}+2}, \ldots, p_{2 k-2} ;
\end{aligned}
$$

and, after $s$ steps, we have

$p_{1}+\sum_{2 k-s+1}^{2 k} p_{\rho}-N, p_{2}, \ldots, p_{l_{s}-1}, p_{l}+p_{l_{s}+1}, p_{l_{s}+2}, \ldots$,

$$
p_{l_{1-1}}, p_{l_{1}}+p_{l_{1}+1}, p_{l_{1}+2}, \ldots, p_{2 k-s},
$$

with the conditions

$$
\begin{aligned}
& \sum_{1}^{l_{1}} p_{\rho}=N, \\
& \sum_{l_{r+1}}^{l_{r-1}} p_{\rho}=p_{2 k-r+2} \quad(r=2,3, \ldots, s) .
\end{aligned}
$$


1908.] Certain periodic properties of cyclic compositions of nUmbers. 281

Hence

$$
\begin{aligned}
p_{1}+\sum_{2 k-8+1}^{2 k} p_{\rho}-N & =p_{2 k-s+1}+p_{1}+\sum_{l_{s+1}}^{l_{1}} p_{\rho}-N \\
& =p_{2 k-s+1}+p_{1}-\sum_{1}^{l_{s}} p_{\rho} \\
& =p_{2 k-s+1}-\sum_{2}^{l_{s}} p_{\rho} .
\end{aligned}
$$

The smallest value of $l_{s}$ is 2 . Let the final value of $s$ be $t$, so that

while

$$
\begin{aligned}
& p_{2 k-t+1}>\sum_{2}^{l_{t}} p_{\rho} \\
& p_{2 k-t+2}<\sum_{2}^{l_{t-1}} p_{\rho} .
\end{aligned}
$$

Then

$p_{2 k}=\sum_{l_{2}+1}^{l_{1}} p_{\rho}, \quad p_{2 k-1}=\sum_{l_{s}+1}^{l_{2}} p_{\rho}, \ldots, \quad p_{2 k-t+2}=\sum_{l_{t+1}}^{l_{t-1}} p_{\rho}, \quad p_{2 k-t+1}>\sum_{2}^{l_{2}} p_{\rho}$.

Now

$$
l_{t}<l_{t-1}<\ldots<l_{2}<l_{1}<k .
$$

Hence $t$ will be $<k-1$ unless

$$
\begin{aligned}
& l_{r+1}=l_{r}-1 \quad(r=1,2, \ldots, t-1), \\
& l_{t}=2 \text {, } \\
& l_{1}=k \text {. } \\
& \text { Hence } t=k-1 \text {, and } \quad l_{r}=k-(r-1) \\
& p_{2 k-r+2}=p_{l_{r-1}}=p_{k-r+2} \text {, } \\
& p_{s}=p_{k+s *}
\end{aligned}
$$

Hence the composition must be regular or an even alien.

21. An odd alien composition of $2 N$ appertaining to $2 m$ reduces, modulo $N$, to an odd alien composition of $N$ appertaining to $m . *$

Let $Q=p_{1}, p_{2}, \ldots, p_{t}$ repeated $2 k+1$ times, or $P^{2 k+1}$, where

$$
\sum_{1}^{t} p_{\rho}=2 m \text { and } N=(2 k+1) m \text {. }
$$

$$
\begin{gathered}
Q(\bmod N)=p_{t}, p_{1}-N, p_{2}, \ldots, p_{t}, P^{2 k-1}, p_{1}, \ldots, p_{t-1} \\
=p_{t}+p_{1}-N, p_{2}, \ldots, p_{r-1}, N-\sum_{1}^{r-1} p_{\rho}, \sum_{1}^{r} p_{\rho}-N, p_{r+1}, \ldots, \\
p_{l}, P^{* k-1}, p_{1}, \ldots, p_{t-1} .
\end{gathered}
$$

\footnotetext{
* This theorem is not esseutial for what follows.
} 
Put $r=t$, and we get

$$
\begin{aligned}
& p_{t}+p_{1}-N, p_{2}, \ldots, p_{t-1}, N-\sum_{1}^{t-1} p_{\rho}, \sum_{1}^{t} p_{\rho}-N, P^{2 k-1}, p_{1}, \ldots, p_{t-1} \\
= & p_{t}+p_{1}-N, p_{2}, \ldots, p_{t-1}, p_{t}, N-2 m, p_{1}-(N-2 m), p_{2}, \ldots, \\
& p_{t}, P^{2 k-2}, p_{1}, \ldots, p_{t-1} .
\end{aligned}
$$

In the same way this becomes

$p_{t}+p_{1}-N, p_{2}, \ldots, p_{t}, P^{r-1}, N-2 r m, p_{1}-(N-2 r m), p_{2}, \ldots$,

and, putting, $r=k$,

$$
p_{t}, P^{2 k-r-1}, p_{1}, \ldots, p_{t-1}
$$

$$
\begin{aligned}
& p_{t}+p_{1}-N, p_{2}, \ldots, p_{t}, P^{k-1}, m, p_{1}-m, p_{2}, \ldots, p_{t}, P^{k-1}, p_{1}, \ldots, p_{t-1} \\
= & p_{t}+p_{1}-N, p_{2}, \ldots, p_{t}, P^{k-1}, m, P(\bmod m), P^{k-1}, p_{1}, \ldots, p_{t-1} .
\end{aligned}
$$

Again, this becomes

$p_{t-1}+p_{t}+p_{1}-1, p_{2}, \ldots, p_{r-1}, N-p_{t}-\sum_{1}^{r-1} p_{\rho}, \sum_{1}^{r} p_{\rho}-N+p_{t}, p_{r+1}, \ldots$,

and, putting $r=t-1$, $p_{t}, P^{k-1}, m, \ldots, p_{t-2}$ $p_{t-1}+p_{t}+p_{1}-N, p_{2}, \ldots$,

$p_{t-2}, p_{t-1}, N-2 m, p_{t}-(N-2 m), P^{k-1}, m, P(\bmod m), P^{k-1}, p_{1}, \ldots, p_{t-1}$, which becomes, as above, $p_{t-1}+p_{t}+p_{1}-N, p_{2}, \ldots, p_{t}, P^{r-2}, p_{1}, \ldots, p_{t-1}, N-2 r m, p_{t}-(N-2 r m)$, and, putting $r=k$,

$$
P^{k-r}, m, P(\bmod m), P^{k-1}, p_{1}, \ldots, p_{t-2},
$$

$p_{t-1}+p_{t}+p_{1}-N, p_{2}, \ldots, p_{t}, P^{i-2}, p_{1}, \ldots, p_{t-1}, m, p_{t}-m, m, P(\bmod m)$,

$$
P^{k-1}, p_{1}, \ldots, p_{t-2}
$$

$=p_{t-1}+p_{t}+p_{1}-N, p_{2}, \ldots, p_{t}, P^{k-1}, m-p_{t}, p_{t}, P(\bmod m), P^{k-1}, p_{1}, \ldots, p_{t-2}$.

In the same way we get

$p_{t-2}+p_{t-1}+p_{t}+p_{1}-N, p_{2}, \ldots, p_{t}, P^{k-1}, m-p_{t-1}-p_{t}, p_{t-1}, p_{t}, P(\bmod m)$,

Proceeding in this way we get

$$
P^{k-1}, p_{1}, \ldots, p_{t-3} \text {. }
$$

$\sum_{t-1}^{\ell} p_{\rho}+p_{1}-N, p_{2}, \ldots, p_{t}, P^{k-1}, m-\sum_{t-r+1}^{l} p_{\rho}, p_{t-r+1}, \ldots, p_{\ell}, P(\bmod m)$,

$$
P^{k-1}, p_{1}, \ldots, p_{t-r-1}
$$


1908.] Certain Periodic PRoperties of CYCLIC COMPOSITIONS OF NUMBERS. 283

and, putting $r=t-1$,

$$
\begin{aligned}
& p_{1}+\sum_{1}^{t} p_{\rho}-N, p_{2}, \ldots, p_{t}, P^{k-1}, m-\sum_{2}^{t} p_{\rho}, p_{2}, \ldots, p_{t}, P(\bmod m), P^{k-1} \\
= & p_{1}-(N-2 m), p_{2}, \ldots, p_{t}, P^{k-1},\{P(\bmod m)\}^{2}, P^{k-1} .
\end{aligned}
$$

By repeating these series of operations we get again

$$
p_{1}-(N-2 r m), p_{2}, \ldots, p_{\ell}, P^{k-r},\{P(\bmod m)\}^{2 r}, P^{k-r},
$$

and; putting $r=k$,

$$
p_{1}-m, p_{2}, \ldots, p_{l},\{P(\bmod m)\}^{2 k}=\{P(\bmod m)\}^{2 k+1},
$$

which proves the theorem.

If $N$ is an even multiple of $m=2 \mathrm{~km}, N-2 \mathrm{rm}$ vanishes when $r=k$, and we get successively

$$
\begin{aligned}
& p_{t}+p_{1}-N, p_{2}, \ldots, p_{t}, P^{k-1}, 0, P^{k-1}, p_{1}, \ldots, p_{t-1} \\
& p_{t-1}+p_{t}+p_{1}-N, p_{2}, \ldots, p_{t}, P^{k-2}, p_{1}, \ldots, p_{t-1}, 0, p_{t}, 0, P^{k-1}, p_{1}, \ldots, p_{t-2} \\
& p_{1}-(N-2 m), p_{2}, \ldots, p_{t}, P^{k-2}, p_{1}, 0, \ldots, p_{t}, 0, P^{k-1},
\end{aligned}
$$

and ultimately every second part becomes zero, and the whole composition condenses to a single part, as in the case, already considered, $\$ 19$, where $k=1$.

\section{Every composition of $2^{m}$ is degenerate.}

Let us assume this to be true for compositions of all powers of 2 up to $2^{m-1}$, and let $P$ be any composition of $2^{m}$. Then $P\left(\bmod 2^{n-1}\right)$ is a composition of $2^{m-1}$, and, by assumption, it finally evolves to 0 . Hence the corresponding evolute of $P$ must be regular, and $P$ therefore degenerates. But all the compositions of 4, viz., 1,3 and 2, 2 degenerate to 0 , hence the result is true generally.

In particular consider the regular composition $2^{r}, 2^{r}$,

$$
2^{r}, 2^{r}=2^{r}(1,1) \quad \text { and } E(1,1)=0 \text {; }
$$

therefore

$$
E^{2^{r}}\left(2^{r}, 2^{r}\right)=0, *
$$

and

$$
E^{2^{r}-1}\left(2^{r}, 2^{r}\right)=2^{r+1} \text { ones. }
$$

- See below, $\$ 25$, II. The equation $E(1,1)=0$ is symbolic. The composition 1,1 denotes a row of + and - alternately, and on evolution gives - repeated indefinitely, and this is represented by $(0) 2$, or, in fact, $(0) x$ where $x$ is any positive integer. Evolving again we get a row of + signs alone or $x(0)$, and repeated evolution does not alter this. 
23. Condition that a composition of $2 N$ should be degenerate.

If $P$ is degenerate $E^{k} P$ is regular for some value of $k$, and

$$
\left.\vdots E^{k} P_{\uparrow}^{\nmid}(\bmod N)=E^{k}{ }_{i} P(\bmod N)\right\}=0 .
$$

Therefore $P(\bmod N)$ is an alien composition of $N$ consisting of a composition of a power of 2 repeated. Conversely, if $P(\bmod N)$ is an alien composition of $N$ appertaining to a power of $2, E^{k} P(\bmod N)$ vanishes, and therefore $E^{k} P$ is regular and $P$ is degenerate.

It may happen that, while $P$ is not degenerate, $P(\bmod N)$ is an alien composition of $N$. Thus, if

$$
P=112116, \quad P(\bmod 6)=112110=1212 ;
$$

but $P$ belongs to a period $(112116,1353,122214,11111232)$. The necessary and sufficient condition that $P$ be degenerate is therefore that $N$ should be a power of 2 , or that $P(\bmod N)$ should be an alien appertaining to a power of 2.

24. Successive Evolution.

Let

$$
P=p_{1}, p_{2}, \ldots, p_{u}
$$

be any partition of $N$. Then

$$
\begin{aligned}
& E P=p_{1}-1,1, p_{2}-1,1, \ldots, \\
& E^{2} P=p_{1}-2,2, p_{2}-2,2, \ldots, \\
& E^{3} P=p_{1}-3,1,1,1, p_{2}-3,1,1,1, \ldots, \\
& E^{4} P=p_{1}-4,4, p_{2}-4,4, \ldots,
\end{aligned}
$$

and, generally, $\quad E^{n} P=p_{1}-n,\left\{n_{i}^{\prime}, p_{2}-n,\{n\}, \ldots\right.$,

where $\{n\}$ is an odd composition of $n$ obeying the law

for $k<2^{n}$.

$$
\left\{2^{m}+k\right\}=\left\{k !, 2^{n}-k,\{k\},\right.
$$

To prove this, assume first that $\left\{2^{m}\right\}=2^{n}$, for $m=r$. Then

$$
E^{2^{r}} P=p_{1}-2^{r}, 2^{r}, p_{2}-2^{r}, \ldots,
$$

and

$$
\begin{aligned}
E^{2^{r+1}} P & =E^{2^{r}} . E^{2^{r}} P=p_{1}-2^{r}-2^{r}, 2^{r}, 0,2^{r}, p_{2}-2^{r}-2^{r}, \ldots \\
& =p_{1}-2^{r+1}, 2^{r+1}, p_{2}-2^{r+1}, \ldots
\end{aligned}
$$


1908.] Certain periodic PRoperties of CYCLIC COMPOStTIONS OF NiJibers. 285

Hence the assumption is established for all values of $m$. Then

$$
E^{2^{m}} P=p_{1}-2^{m}, 2^{m}, p_{2}-2^{n}, \ldots,
$$

and $\left.E^{2^{m}+k} P=E^{k} \cdot E^{2^{m}} P=p_{1}-2^{m}-k, \vdots k\right\}, 2^{n k}-\dot{\kappa}, \vdots k_{i}^{\prime}, p_{2}-2^{i n}-k, \ldots$

$$
=p_{1}-2^{m}-k,\left\{2^{m}+k\right\}, p_{2}-2^{m}-k, \ldots
$$

Hence

$$
\left\{2^{m}+k\right\}=\{k\}, 2^{m}-k,\{k ; .
$$

Now, if for the sake of brevity we write $[r]$ instead of

$$
2^{m_{k-r+1}}-2^{m_{k-r+2}}-\ldots-2^{m_{k}},
$$

then $\left\{2^{m_{1}}+2^{m_{2}}+\ldots+2^{m_{k}}\right\}$

$$
\begin{array}{r}
=[1][2][1] \ldots[1][2][1][3][1][2][1][k][1][2][1][3][1][2][1][t] \\
\ldots[1][2][1][k-1][1] \ldots[1][2][1] .
\end{array}
$$

It thus contains $2^{k}-1$ parts.

The form of $\{n\}$ can be rapidly found by working in the scale of 2 . To find $[k]$ subtract $n$ from the next power of 2 ; to find $[k-1]$ strike off the first significant figure from $n$ on the left and subtract from the next power of 2, and so on. Thus, if $n=85$, or, in the scale of 2, 1010101;

$$
[4]=101011=43,[3]=1011=11,[2]=11=3,[1]=1 \text {; }
$$

and $\quad\{85\}=1,3,1,11,1,3,1,43,1,3,1,11,1,3,1$.

The process of eliminating negative parts is much more laborious than evolution, so that often the quickest way to obtain any evolute of a composition is to evolve repeatedly.

25. The operation of doubling a composition.

Let $P$ be a composition of $N$, and let each of its parts be doubled. Then we get a composition of $2 N$ in which every part is eren. Denote this by $2 P$.

THEOREM I.- $\quad E^{2}(2 P)=2 E(P)$.

Let

$$
P=p, q, 1^{r}, s, \ldots \text {. }
$$

Then

$$
\begin{aligned}
E P & =p-1,1, q-1, r+1, s-1, \ldots, \\
2 P & =2 p, 2 q, r \text { twos, } 2 s, \ldots, \\
E .2 P & =2 p-1,1,2 q-1,2 r+1 \text { ones, } 2 s-1, \ldots, \\
E^{2} .2 P & =2 p-2,2,2 q-2,2 r+2,2 s-2, \ldots \\
& =2 . E P .
\end{aligned}
$$


Theorem II.- $\quad E^{2^{r}}\left(2^{r} P\right)=2^{r} . E(P)$.

Assume this to be true for $r$. Then

$$
\begin{aligned}
E^{2^{r+1}}\left(2^{r+1} P\right) & =E^{2^{r}}\left\{E^{2^{r}}\left(2^{r+1} P\right)\right\} \\
& =E^{2^{r}}\left\{2^{r} E(2 P)\right\}=2^{r} . E(E \cdot 2 P) \\
& =2^{r} \cdot E^{2}(2 P)=2^{r} \cdot 2 \cdot E P \\
& =2^{r+1} \cdot E(P) .
\end{aligned}
$$

Hence the theorem is true generally.

Theorem III.-

$$
2^{r} . E^{s} P=E^{s .2^{r}} .2^{r} P .
$$

Assume this for $s$. Then

$$
\begin{aligned}
2^{r} E^{s+1} P & =E^{s .2^{r}} \cdot 2^{r} \cdot E P \\
& =E^{s . \vartheta^{r}} \cdot E^{2^{r}} \cdot 2^{r} P \\
& =E^{(s+1) \varkappa^{r}} \cdot 2^{r} P .
\end{aligned}
$$

Corollaries.-Every alternate evolute of $2 P$ has all its parts even.

If $P$ belongs to a period of $n, 2 P$ will helong to a period of $2 n$.

If the $r$-th evolute of $P$ is pure, the $2 r$-th evolute of $2 P$ will be pure.

If $P$ is regular, $2 P$ is also regular, and if the $r$-th evolute of $P$ is regular, the $2 r$-th evolute of $2 P$ will be regular; then the $(r+1)$-th evolute of $P$ is alien and the $(2 r+1)$-th evolute of $2 P$ will be alien.

\section{Doubling a composition and reducing modulo $N$.}

Let the combined operation of doubling a composition of $N$, and at the same time reducing modulo $N$, be denoted by $D$, so that

$$
D P=2 P(\bmod N) .
$$

Then $D P$ is also a composition of $N$. In this way we can pass from one composition to another composition of the same number.

Theorems of exactly the same form as I. to III. of the preceding section can be proved in the same way, only reducing the final results modulo $N$. It is unnecessary to state the theorems, as we have simply to substitute $D$ for 2 in the operator (not in the index).

If $N$ is odd, DP and $P$ contain the same number of parts.

In reducing $p_{1}, p_{2}, \ldots$, modulo $N$, the parts are always of the form $p_{r}$ or $\pm\left(p_{r}+p_{s}+\ldots-N\right)$. Hence, since $p_{r}$ is always even and $N$ is odd, this can never vanish and condensation never occurs.

If $N$ is even, condensation may occur, and $D P$ has all its parts even, for all the numbers which occur at any stage are even. 
27. The reverse process.

First introduce zero or negative parts and transform by the usual rules until there is not more than one zero or negative part, and (1) if $N$ is even there are no odd parts, (2) if $N$ is odd, only one odd part which may be positive or negative. Then add $N$ to the zero, negative or odd part, or to any part if all the parts are even, and divide each part by 2 .

If $N$ is even the reverse process is possible only if all the parts are even, and is evidently not unique, for from $2 p, 2 q, 2 r, 2 s$ we get, e.g., $p+\frac{1}{3} N, q, r, s$ or $p, q+\frac{1}{2} N, r, s$ or $p_{1}, \frac{1}{2} N, p_{2}, q, r, s$, and so on, where

$$
p_{1}+p_{2}=p \text {. }
$$

If $N$. is odd the reverse process is always possible and it is unique.

To prove this, we notice that the only transformation available is

$$
p, q, r, s=p, q+r, \bar{r}, r+s,
$$

since the number of parts must remain the same ( $\$ 26)$.

Since $N$ is odd there must be an odd number of odd parts. If three odd parts come together they may be reduced to one, for, distinguishing an odd number by enclosing it within brackets,

$$
(p),(q),(r), \ldots=p+q,(\bar{q}), r+q, \ldots
$$

If two odd parts come together they may, by a succession of steps, be transferred to any other position in the composition.

For,

$(p),(q), r, s, \ldots=p+q,(\bar{q}),(r+q), s, \ldots=p+q, r,(\overline{r+q}),(q+r+s), \ldots$, and so on.

If one odd part occurs by itself, it may be made first to give rise to three, thus

$$
p,(q), r, s, \ldots=(p+q),(\bar{q}),(q+r), s, \ldots .
$$

Then either of the pairs which occur together may be moved along the series and the single odd part, say $(p+q)$, has been moved one place in the opposite direction, as

$$
(p+q), r, \overline{(q+r)},(q+r+s), \ldots .
$$

The following process then suffices to eliminate all the odd parts except one. First eliminate all triplets. Then move any pair of odd parts until it comes adjacent to another odd part, producing a triplet which reduces to a single odd part. We are now left with an odd number of isolated odd parts. Suppose there are three odd parts, in positions numbered $1, m, n$. Transform the middle one into a triplet and move the pair on the right until it forms a triplet with the third part and eliminate 
this triplet. We now have three odd parts in positions $1, m-1, n-1$. Treating the middle one again in the same way, we get three odd parts in positions $1, m-2, n-2$. Proceeding in this way, we get at last the three odd parts in positions $1,2, n-m+2$. Then the pair can be moved to the right until it forms a triplet with the third part, and this then reduces to a single odd part. By a repetition of this process we shall finally be left with a single odd part which may be positive or negative, the even parts at any stage being always positive. Then, adding $N$ to this part and dividing all through by 2 , we get the required composition.

Now these compositions, in whatever order the processes are performed, are all equivalent, modulo $N$, and the final result before adding $N$ is always the same. Hence, as there is only one way in which the $N$ may be added, the whole process is unique.

In the following sections 28-30 we shall suppose $N$ to be odd.

28. If a composition of $N$ be multiplied repeatedly by 2 , only reducing modulo $N$ when it is possible without introducing negative parts, it will return to itself after $p$ multiplications and not before, where $p$ is the $\theta x$ ponent to which 2 appertains $(\bmod N)$.

For, since $\quad 2^{p} \equiv 1(\bmod N), \quad x .2^{p} \equiv x(\bmod N)$,

and, without the introduction of negative parts, it cannot return to its original value before this.

Hence, for any composition $P$ of $N$,

$$
D^{p} P=P .
$$

Again, if there exists an index $t$, such that $2^{t} \equiv-1(\bmod N)$, the composition obtained after $t$ multiplications, when reduced repeatedly, modulo $N$, until the sum of its parts is equal to $N$, is the same as the original composition, but written in the reverse order.

For, since $2^{t} \equiv-1(\bmod N), \quad x .2^{t} \equiv-x(\bmod N)$,

therefore the composition obtained from

$$
p_{1}, p_{2}, \ldots, p_{k},
$$

after $t$ multiplications, can be written

$$
\begin{gathered}
\bar{p}_{1}, \bar{p}_{2}, \ldots, \bar{p}_{k}, \\
\text { or, making the sum of the parts }=N=\sum_{1}^{k} p_{\rho}, \\
\bar{p}_{1}, \bar{p}_{2}, \ldots, \bar{p}_{k-2}, N-p_{k-1}, N-p_{k} .
\end{gathered}
$$


1908.] Certain periodic Properties of CYCLIC Compositions of NUMBers. 289

This is equal to

$$
\begin{aligned}
& p_{1},-p_{1}-p_{2},-p_{3}, \ldots,-p_{k-2}, N-p_{k-1}, N-p_{k}-p_{1} \\
= & -p_{2}, p_{1}+p_{2},-p_{1}-p_{2}-p_{3},-p_{4}, \ldots \\
= & \bar{p}_{2}, \bar{p}_{3}, \ldots, \bar{p}_{r-1}, \sum_{1}^{r-1} p_{\rho},-\sum_{1}^{r} p_{\rho}, \bar{p}_{r+1}, \ldots \\
= & \bar{p}_{2}, \bar{p}_{3}, \ldots, \bar{p}_{k-3}, \sum_{1}^{k-3} p_{\rho},-\sum_{1}^{k-2} p_{\rho}, N-p_{k-1}, N-p_{k}-p_{1} \\
= & \bar{p}_{2}, \bar{p}_{3}, \ldots, \bar{p}_{k-2}, \sum_{1}^{k-2} p_{\rho}, N-\sum_{1}^{k-1} p_{\rho}, N-p_{k}-p_{1} \\
= & \bar{p}_{2}, \bar{p}_{3}, \ldots, \bar{p}_{k-2}, \sum_{1}^{k-2} p_{\rho}, p_{k}, N-p_{k}-p_{1} .
\end{aligned}
$$

In the same way this becomes

$$
\begin{aligned}
& \bar{p}_{3}, \ldots, \bar{p}_{r-1},{ }_{2}^{r-1} p_{\rho},-\sum_{2}^{r} p_{\rho}, \bar{p}_{r+1}, \ldots \\
= & \bar{p}_{8}, \ldots, \bar{p}_{k-3},{ }_{2}^{k-3} p_{\rho},-\sum_{2}^{k-2} p_{\rho}, \sum_{1}^{k-2} p_{\rho}, p_{k}, N-p_{k}-p_{1}-p_{2} \\
= & \bar{p}_{3}, \ldots, \bar{p}_{k-2}, \sum_{2}^{k-2} p_{\rho}, p_{1}, p_{k}, N-p_{k}-p_{1}-p_{2} ;
\end{aligned}
$$

and, similarly,

$$
\begin{aligned}
& \bar{p}_{r}, \ldots, \bar{p}_{k-2}, \sum_{r-1}^{k-2} p_{\rho}, p_{r-2}, p_{r-3}, \ldots, p_{1}, p_{k}, N-p_{k}-\sum_{1}^{r-1} p_{\rho} \\
= & \bar{p}_{k-2}, \sum_{k-3}^{k-2} p_{\rho}, p_{k-4}, p_{k-5}, \ldots, p_{1}, p_{l}, N-p_{k}-\sum_{1}^{k-3} p_{\rho} \\
= & p_{k-2}, p_{k-3}, \ldots, p_{1}, p_{k}, p_{k-1} .
\end{aligned}
$$

Hence, if $N$ is such that $2^{t} \equiv-1(\bmod N)$, and therefore $2^{2 t} \equiv 1(\bmod N)$, every composition when multiplied $t$ times by 2 and reduced modulo $N$, gives the enantiomorphic composition, and if it is symmetrical it returns to itself.

It may happen that when a composition is multiplied by 2 repeatedly, and reduced modulo $N$ each time, it will return to itself or give its image after fewer multiplications than $t$. If it returns after $s$, so that

then also

$$
D^{2} P=P \text { or } P^{\prime} \text {, }
$$

and $s$ must be a factor of $t$. 
Also it may return to its image after $s$ doublings, although there is no number $t$ such that $2^{t} \equiv-1(\bmod N)$. In this case, however, as in the other, $s$ is equal to or a factor of $\frac{1}{2} P$. E.g., 1455 gives 2855 , and then 4155 ; but $p=4$, and no number $t$ exists for which $2^{t} \equiv-1(\bmod 15)$. Such an occurrence is evidently dependent upon the form of the composition.

29. If $P$ belongs to a period, $D P$ will also belong to a period, which may be the same period or another; corresponding members of the new period are not in the order of evolution, but each one is the second evolute of the preceding.

Let $D P=P_{1}, D P_{1}=P_{2}, \ldots$, then $P_{s}=P$, where $s$ is equal to or a factor of $p$, the smallest index for which $2^{p} \equiv 1(\bmod N)$, and we have a set of $s$ compositions, $P, P_{1}, P_{2}, \ldots, P_{s-1}$, all with the same number of parts. Now, if $P$ belongs to a period of $n$, then since no two different compositions give the same result when doubled and reduced, $P_{1}$ will belong to a period of $n$ or $2 n$. In the same way $P_{2}$ will belong to a period of $n, 2 n$ or $4 n$, and the period of $P_{s-1}$ will consist of $2^{r}$. n, where $r<s-1$. Since $P_{s-1}$ now returns to $P, P$ 's period would contain $2^{r} . n(r \gtrless s)$, but it contains $n$; hence each of the periods must contain only $n$. The $n$ members of $P$ 's period must therefore, when doubled, give all the members of the period of $P$, and these occur in alternate order, hence $n$ must be odd.

We have seen that, if a period contains both of a pair of enantiomorphs, each composition will be accompanied by its image, and the number of members in the period would therefore be even. When $N$ is odd this is therefore impossible and enantiomorphic periods occur in pairs. Such periods are primarily isomorphic, i.e., to each composition with $a$ parts in one period corresponds a composition with a parts in the other, and these occupy the same relative positions in the two periods.

30. Let us now examine more carefully the periods generated by $P, P_{1}, \ldots, P_{s-1}$. Let the first period be represented by

$$
(0,1,2,3, \ldots, n-1) \text {. }
$$

Let 0 become $0_{1}, 1$ become $1_{1}, \ldots$, then the second period is

$$
\left\{0_{1},\left(\frac{n+1}{2}\right)_{1}, 1_{1},\left(\frac{n+3}{2}\right)_{1}, 2_{1}, \ldots,(n-1)_{1},\left(\frac{n-1}{2}\right)_{1}\right\} \text {. }
$$

$n$ is of course odd. Let the symbols of the members of the new period be permuted cyclically and placed below the original period. Then if $0_{1}$ falls under $r, 1_{1}$ will fall under $r+2, \ldots, s_{1}$ will fall under $r+2 s$. Hence 
1908.] Certain periodic properties of CyCLic conipositions of ncibers. 291

the pair of periods represent a substitution which will contain cycles of the form

$$
\begin{array}{ll}
\text { the form } & 1, r, r+2 r, r+2(r+2 r), \ldots, \\
\text { i.e., } & 1, r, 3 r, 7 r, \ldots,\left(2^{p}-1\right) r, \ldots
\end{array}
$$

Suppose a cycle to contain $p$, then

$$
\left(2^{i}-1\right) r \equiv 1(\bmod n) \text {. }
$$

If $r$ is prime to $n$, this can only be satisfied if

$$
2^{p} \equiv 1(\bmod n)
$$

Again, we shall have a cycle

$$
s, r+2 s, 3 r+4 s, \ldots,\left(2^{i}-1\right) r+2^{p} s, \ldots,
$$

and, if

$$
\begin{aligned}
\left(2^{p}-1\right) r+2^{p} s & \equiv s(\bmod n), \\
\left(2^{p}-1\right)(r+s) & \equiv 0(\bmod n),
\end{aligned}
$$

which, if $r+s$ is prime to $n$, is only possible if

$$
2^{p} \equiv 1(\bmod n) \text {. }
$$

If $r+s=n$, i.e., $s=n-r, s$ forms a cycle of 1 . Hence

If the periods generated by $P$ and $D P$ are the same there is one mem. ber of the period, $A$, such that $D A=A$.

If $D P$ does not belong to the period of $P$ it will belong to another period, and these two periods will be called secondarily isomorphic, i.e., if we consider only the number of parts in the compositions, and $P$ 's period is denoted by

$$
(a b c \ldots) \text {, }
$$

where, of course, some of the letters are in general the same, then considering this as representing a substitution, the period of $D P$ will be represented by

$$
(a b c \ldots)^{2} \text {. }
$$

When the two periods are the same a secondary isomorphism is estabiished within the period.

The set of periods generated by $P, P_{1}, \ldots, P_{s-1}$ will be called a conjugate set; and when the periods generated by $P$ and $D P$ are the same the period will be said to be self-conjugate.

If there is an index $t$, such that $2^{t} \equiv-1(\bmod N)$, then $D^{t} P=P$, if $P$ is symmetrical, and $=P^{\prime}$ if $P$ is not symmetrical. In the first case the conjugate sets contain $t$ or a factor of $t$; in the second case they contain $2 t$ or an even factor of $2 t$, and each set contains pairs of enantiomorphic periods. 
If there is no such number $t$, and $2^{p} \equiv 1(\bmod N)$, the number of periods in a conjugate set is $p$ or a factor of $p$, and the enantiomorphic pairs of periods may occur in the same set if $p$ is even.

When $P$ is symmetrical the set of periods to which it belongs is a symmetrical conjugate set.

When $P$ is not symmetrical and the conjugate set does not contain both of a pair of enantiomorphs, we have a pair of enantiomorphic conjugate sets. If there is no number $t$ such that $2^{t} \equiv-1(\bmod N)$, we may have a pair of enantiomorphic self-conjugate periods.

When $P$ is not symmetrical and the conjugate set contains both of a pair of enantiomorphs there is one conjugate set to which both $P$ and $P^{\prime}$ belong. Such a set will be called racemic.

31. The following theorems will be required in what follows.

$$
\begin{aligned}
\text { Theorem I. } & -p_{1}-x, x, p_{2}-x, x, \ldots, p_{a}-x, x \\
& =p_{1}-x+k N, x-k N, p_{2}-x+k N, x-k N, \ldots,
\end{aligned}
$$

where

$$
N=\Sigma p \text {. }
$$

We have

$$
p_{1}-x, x, p_{2}-x, \ldots
$$

$=x-p_{1}, p_{1}+p_{2}-x, x-p_{2}, p_{2}+p_{3}-x, \ldots$

$=p_{a}+p_{1}+p_{2}-x, x-\left(p_{1}+p_{2}\right), p_{1}+p_{2}+p_{3}-x, x-\left(p_{2}+p_{3}\right), \ldots$

$=\sum_{1}^{r} p_{\rho}-x, x-\sum_{2}^{r} p_{\rho}, \sum_{2}^{r+1} p_{\rho}-x, x-\sum_{3}^{r+1} p_{\rho}, \ldots$

Putting $r=a$, we get

$$
\begin{aligned}
& N-x, x-N+p_{1}, N-x, x-N+p_{2}, \ldots \\
= & x-N, p_{1}-x+N, x-N, p_{2}-x+N, \ldots .
\end{aligned}
$$

Now assume the theorem for $k$, then

$$
\begin{aligned}
& p_{1}-x+k N, x-k N, p_{2}-x+k N, x-k N, \ldots \\
= & p_{1}-x+(k+1) N, x-(k+1) N, \ldots
\end{aligned}
$$

Hence the theorem is established.

Theoren II. $-1^{p},-x+1,1^{4}, \ldots=1^{p-x}, x+1,1^{q-x}, \ldots$

We have $1^{p},-x+1,1^{4}, \ldots$

$$
\begin{aligned}
& =1^{p-1},-x+2, x-1,-x+2,1^{q-1}, \ldots \\
& =1^{p-2},-x+3, x-2,-x+3, x-2,-x+3,1^{1-2}, \ldots \\
& =1^{p-k},(-x+k+1, x-k)^{k},-x+k+1,1^{q-k}, \ldots
\end{aligned}
$$


1908.] Certain periodic properties of Cyclic conpositions of ncuibers. 293

Let $k=x$, and we have

$$
\begin{aligned}
& 1^{p-j},(1,0)^{x}, 1,1^{1-x}, \ldots \\
= & 1^{p-x}, x+1,1^{1-x}, \ldots
\end{aligned}
$$

32. We proceed now to investigate the number of compositions in a period, first in the case where $N$ is odd.

Suppose first that $A$ is a member of a period such that $D A=A$. Then, if $E A=B$,

$$
\begin{gathered}
E B=E^{2} A=E^{2} D A=D E A=D B, \\
E^{2^{r}} D^{r} B=D^{r} E B=D^{\prime} D B=D^{r+1} B, \\
E^{2^{r-1}} D^{r-1} B=D^{r} B, \\
\cdots \quad \cdots \quad \cdots \quad \cdots \\
\quad D^{r+1} B=E^{r 2^{r}} B=E^{2^{r+1}-1} B .
\end{gathered}
$$

Hence

But, if $2^{t} \equiv \pm 1(\bmod N), \quad D^{t} B=B$,

hence

$$
E^{2^{t}-1} B=B \text {. }
$$

[If $2^{t} \equiv-1(\bmod N) A$ must, of course, be symmetrical.]

Second, if $D A_{0}$ is not a member of the same period as $A_{0}$, we have a set of isomorphic periods generated by $A_{0}, D A_{0}=A_{1}, D^{2} A_{0}=A_{2}, \ldots$, and we have $D^{r} A_{s}=A_{r+s}$, where the subscript of $A$ is to be reduced modulo $p, p$ being the exponent to which 2 appertains $(\bmod N)$.

Let $E A_{s}=B_{s}$, then

$$
E^{2^{r}} D^{r} A_{s}=D^{r} E A_{s}=D^{r} B_{s}=E^{2^{r}} A_{s+r},
$$

i.e.,

$$
E^{2^{r}} A_{k}=D^{r} B_{k-r}
$$

Hence

$$
E^{y^{r}} D^{r} B_{s}=E^{2^{r}} E^{2^{r}} A_{s+r}=E^{2^{r-1}} A_{s+r}=D^{r+1} B_{s-1} \text {. }
$$

Putting in succession $\quad r=r, r-1, \ldots, 0$,

$$
s=1,2, \ldots, r+1,
$$

we get

$$
D^{x+1} B_{0}=E^{\Sigma_{0}^{r} 2^{u}} B_{r+1}=E^{2^{r+1}-1} B_{r+1} \text {. }
$$

If $2^{t} \equiv+1(\bmod N)$,

$$
D^{t} B_{0}=B_{0} \quad \text { and } \quad B_{\imath}=B_{0}
$$

therefore

$$
E^{2^{t}-1} B_{0}=B_{0} \text {. }
$$


If $2^{\imath} \equiv-1(\bmod N)$,

$$
D^{t} B_{0}=B_{0}^{\prime} \quad \text { and } \quad B_{t}=B_{0}^{\prime}
$$

therefore

$$
E^{2^{t}-1} B_{0}^{\prime}=B_{0}^{\prime} \text {. }
$$

Hence every composition of $N$ satisfies the equation

$$
E^{2^{2}-1} P=P \text {. }
$$

It may happen, however, that $D^{s} P=P$ or $P^{\prime}$, where $s$ is a factor of $t$, and if every member of a period satisfies this equation, then for every member of this period

$$
E^{2^{6}-1} P=P \text {. }
$$

The period of a composition of $N$ is therefore, in general, $2^{l}-1$, where $t$ is the smallest integer satisfying the congruence $2^{t} \equiv \pm 1(\bmod N)$. We shall call this the normal period of $N$.

In exceptional circumstances there may be short periods, factors of $2^{t}-1$. E.g., 17 has a normal period of 15 , but it has also periods of 5 . (See Appendix. It is noteworthy that there are periods not of the form $2^{n}-1 . \quad 17$ is the smallest number for which such periods exist.)

33. The result $E^{0^{t}-1} P=P$ can be proved more simply thus. Let

$$
P=p_{1}, p_{2}, p_{3}, \ldots
$$

Then

$$
\begin{aligned}
E^{2^{r}} P & =p_{1}-2^{r}, 2^{r}, p_{2}-2^{r}, 2^{r}, \ldots(\$ 24) \\
& =p_{1}-2^{r}+k N, 2^{r}-k N, \ldots(\$ 31, \text { I.). }
\end{aligned}
$$

Now choose $r=t$, so that $2^{t} \equiv \pm 1(\bmod N)$, and $2^{t}=k N \pm 1$. Then we have

$$
\begin{aligned}
& p_{1} \mp 1, \pm 1, \ldots \\
= & p_{1}-1,1, \ldots \text { for either sign } \\
= & E P .
\end{aligned}
$$

Hence

$$
E^{2 t} P=E P \quad \text { and } \quad E^{2^{t}-1} P=P .
$$

This proof, though simpler than that of the last section, does not illustrate the occurrence of short periods of the form $2^{s}-1$.

34. Short periods.

If $s$ is a factor of $t$ there may be a short period of $2^{s}-1$. In particular, even if $t$ is prime, there may be short periods of 1 . A short period of 1 can only be obtained in the following way. Take a composition of $M$, 
1908.] Certain Periodic Properties of CrClic compositions of numbers. 295

say $P$, form its first evolute $E P$, where if $p$ is the first part of $P, p-1$ is the first part of $E P$. Form the successive evolutes in the same way, and place them consecutively, and we get

$$
\begin{aligned}
Q & =P, E P, E^{2} P, \ldots, \\
E Q & =E P, E^{2} P, E^{8} P, \ldots,
\end{aligned}
$$

so that if the series recurs $E Q=Q$.

In this general process negative and zero parts will, 'of course, arise, and in the general case it is not possible to say whether or at what point the series will recur, without having a method of eliminating zero and negative parts.

Two cases, however, may be noted.

(1) Let

$$
P=2^{r} \text {. }
$$

Then we get

$2^{r} ; 2^{r}-1,1 ; 2^{r}-2,2 ; \ldots ; 2^{r}-2^{k}, 2^{k} ; \ldots ; 2^{r-2^{r-1}}, 2^{r-1} ; \ldots ;$

$$
2^{r}-2^{r}+1,2^{r}-1 \text { ones, } 0,2^{r}, \overline{1}, \ldots
$$

which gives $\quad 2^{r} ; 2^{r}-1,1 ; \ldots ; 2^{r}-1$ ones.

$\left(2^{r}-2^{r}+1,2^{r}-1\right.$ ones) is the $\left(2^{r}-1\right)$-th evolute of $2^{r}$, and

$$
N=2^{r} \cdot 2^{r}-1=2^{2 r}-1 .
$$

Examples of this are 21, 43122111.

(2) Let

$$
P=2^{r}+1 .
$$

Then we get

$2^{r}+1 ; 2^{r}, 1 ; 2^{r}-1,2 ; \ldots ; 2^{r}-2^{r-1}, 1,2^{r-1}-1,1 ; \ldots ; 2,2^{r}-1$ ones $; 1$ and

$$
N=2^{r}\left(2^{r}+1\right)+1=2^{2 r}+2^{r}+1 .
$$

Examples of this are 3211, 5413221111.

35. Now take the case where $N=2^{m} \lambda$.

Let

Then

$$
P=p_{1}, p_{2}, \ldots, p_{a} .
$$

$$
\begin{aligned}
E^{2^{m}} P & =p_{1}-2^{m}, 2^{m}, p_{2}-2^{m}, 2^{m}, \ldots \\
E^{2^{m+t}} P & =p_{1}-2^{m+t}, 2^{m+t}, \ldots \\
& =p_{1}-2^{m+t}+k N, 2^{m+t}-k N, \ldots
\end{aligned}
$$


Choose $k$ so that $2^{t}=k \lambda \pm 1$, then we have

and

$$
2^{m+t}-k N=2^{m}\left(2^{t}-k \lambda\right)= \pm 2^{m},
$$

Hence, if $Q=E^{m} P, \quad E^{2^{m}\left(2^{t}-1\right)} Q=Q$,

and $Q$ belongs to a period of $2^{n}\left(2^{t}-1\right)$ or a factor of this. ${ }^{*}$ The $2^{n}$-th evolute of any composition of $2^{m} \lambda$ is therefore pure, and its normal period is $2^{m}\left(2^{t}-1\right)$.

We shall now show that the $\left(2^{m}-1\right)$-th is the first pure evolute of an improper composition of $2^{m} \lambda$.

Any improper composition may be written

$$
P=p_{1}-1,1, p_{2}-1,1, \ldots, p_{a}-1,1,
$$

where $a$ is odd. Then

$$
E^{2^{m}-1} P=E^{2^{m+t}-1} P=p_{1}-2^{m}, 2^{m}, \ldots=E^{2^{\prime \prime \prime}\left(2^{t}-1\right)} \cdot E^{2^{m}-1} P .
$$

Now we have to show that the $\left(2^{n}-2\right)$-th evolute of $P$, where $P$ is improper, is equal to the first involute, not belonging to the period, of $E^{2^{m}\left(2^{t}-1\right)} E^{2^{m}-1} P$, i.e., of $E^{2^{m+t}-1} P$, or that

$$
\begin{aligned}
& p_{1}-2^{m}+1,2^{m}-1 \text { ones, } p_{2}-2^{m}+1,2^{m}-1 \text { ones, } \ldots \\
= & p_{1}-2^{m+t}-1 \text { ones, } 2^{m+t}+1, p_{2}-2^{m+t}-1 \text { ones, } 2^{m+t}+1, \ldots,
\end{aligned}
$$

where $\sum_{1}^{a} p_{\rho}=N=2^{m} \lambda$ and $a$ is odd.

The right-hand side

$=2^{m+t}-p_{1}-1$ ones, $p_{1}+p_{2}-2^{m+t}+1,2^{m+t}-p_{2}-1$ ones,

$$
\begin{array}{r}
p_{2}+p_{3}-2^{m+t}+1, \ldots \\
=p_{a}+p_{1}+p_{2}-2^{m+t}-1 \text { ones, } 2^{m+t}-p_{1}-p_{2}+1, p_{1}+p_{2}+p_{3}-2^{m+t}-1 \text { ones, } \\
2^{m+t}-p_{2}-p_{3}+1, \ldots \\
=2^{m+t}-p_{a}-p_{1}-p_{2}-1 \text { ones, } p_{a}+p_{1}+p_{2}+p_{3}-2^{m+t}+1, \\
2^{m+t}-p_{1}-p_{2}-p_{3}-1 \text { ones, } p_{1}+p_{2}+p_{3}+p_{4}-2^{m+t}+1, \ldots,
\end{array}
$$

by alternate application of $\S 14$ and $\S 31$, II.

Continuing in this way we get, since $a$ is odd, $2^{m+l}-N-1$ ones, $N+p_{1}-2^{m+t}+1,2^{m+t}-N-1$ ones, $N+p_{2}-2^{m+t}+1, \ldots$

- A proof is still wanting for the theorem (if it is true) that the periods of compositions of $2^{m} \lambda$ are all of the form $2^{m} p$, where $p$ is a period of a composition of $\lambda$; also for the general case of the theorem (true when $N$ is odd) that pairs of enantiomorphs cannot occur in the same period. 
1908.] Certain Periodic Properties of CyClic COMPositions OF NUMBers. 297

Proceeding again in the same way we get

$$
\begin{aligned}
& N-2^{m+t}+p_{a}+p_{1}-1 \text { ones, } 2^{m+t}-N-p_{1}+1 \text {, } \\
& N-2^{m+t}+p_{1}+p_{2}-1 \text { ones, } 2^{m+t}-N-p_{2}+1, \ldots \\
& =2^{m+t}-N-p_{a}-p_{1}-1 \text { ones, } N-2^{m+t}+p_{a}+p_{1}+p_{2}+1 \text {, } \\
& 2^{m+t}-N-p_{1}-p_{2}-1 \text { ones, } N-2^{m+t}+p_{1}+p_{2}+p_{3}+1, \ldots \\
& =2^{m+t}-2 N+p_{1}-1 \text { ones, } 2 N-2^{m+t}+1,2^{m+t}-2 N+p_{2}-1 \text { ones, } \\
& 2 N-2^{m+1}+1, \ldots \\
& =2 N-2^{m+t}+p_{1}-1 \text { ones, } 2^{m+t}-2 N+1,2 N-2^{m \div t}+p_{2}-1 \text { ones, } \\
& =2^{n+t}-2 N-p_{1}-1 \text { ones, } 2 N-2^{m+t}+p_{1}+p_{2}+1 \text {, } \\
& 2^{m+i}-2 N-p_{2}-1 \text { ones, } \ldots \\
& =2^{m+t}-3 N-1 \text { ones, } 3 N-2^{m+t}+p_{1}+1,2^{m+t}-3 N-1 \text { ones, } \\
& 3 N-2^{m+t}+p_{2}+1, \ldots
\end{aligned}
$$

and, for any odd value of $r$,

$$
2^{m+t}-r N-1 \text { ones, } r N-2^{n+t}+p_{1}+1, \ldots
$$

Now

$$
2^{m+t}-r N=2^{m}\left(2^{t}-r \lambda\right)= \pm 2^{n}
$$

by properly choosing $r$.

If

$$
2^{t} \equiv+1 \quad(\bmod \lambda)
$$

we get $\quad 2^{n}-1$ ones, $p_{1}-2^{m}+1,2^{m}-1$ ones, $p_{2}-2^{m}+1, \ldots$

If

$$
2^{t} \equiv-1 \quad(\bmod \lambda) \text {, }
$$

we get

$$
-2^{m}-1 \text { ones, } p_{1}+2^{m}+1,-2^{m}-1 \text { ones, } \ldots
$$

$$
=2^{\text {n }}-1 \text { ones, } p_{1}-2^{\text {in }}+1,2^{m}-1 \text { ones, } \ldots \text {. }
$$

Hence the theorem is established, and we have the result :

The $r$-th evolute of an improper composition of $2^{m} \lambda$ is pure only if $r \varangle 2^{m}-1$, and, conversely, a pure composition of $2^{m} \lambda$ can be involved $2^{m}-1$ times until it becomes improper. The number of impure involutes of a pure composition is $\sum_{0}^{2^{m}-2} 2^{\mu}=2^{2^{m}-1}-1$.

36. Now let $R$ be an improper composition of $2^{m} \lambda$. Then $R\left(\bmod \frac{1}{2} N\right)$ is improper and its successive evolutes are impure until $E^{2^{m-1}-1} R\left(\bmod \frac{1}{2} N\right)$ which is pure, and all the successive evolutes after this are pure. Let $P=E^{2^{\prime \prime}-1} R$; then $P$ is pure and, if $Q$ is any $r$-th involute of $P$, $\left(r<2^{m-1}\right), Q\left(\bmod \frac{1}{2} N\right)$ is equal to the pure $r$-th involute of $P\left(\bmod \frac{1}{2} N\right)$, i.e., all the $r$-th involutes of a pure composition of $2^{m} \lambda$, for $r<2^{m-1}$, reduce, modulo $2^{m-1} \lambda$, to the same pure composition of $2^{m-1} \lambda$. 
37. Next consider an improper composition, $P$, of $2^{n} \lambda$ which evolves to a regular composition, $R$, and then degenerates.

$R(\bmod N)$ vanishes and $E^{k} P\left(\bmod \frac{1}{2} N\right)$ is an alien appertaining to a power of 2 . (\$23.)

Now, if $P$ is an improper composition of $2^{m}, E^{2^{m}-1} P=0$, while $E^{2^{m+\prime}-2} P=1,1, \ldots$ To prove this assume the result for $m$, and let $Q$ be an improper composition of $2^{m+1}$. Then $Q\left(\bmod 2^{m}\right)$ is an improper com. position of $2^{m}$, and $E^{2^{\prime m}-1} Q\left(\bmod 2^{m}\right)=0$. Therefore $E^{2^{m}-1} Q$ is regular, and $E^{2^{m}} Q$ is an improper composition of $2^{m}$ repeated. Hence

$$
E^{2^{2 \prime \prime}-1} \cdot E^{2^{m}} Q=E^{2^{m+1}-1} Q=0 .
$$

But $E(1,1)=0$, bence the result is established.

We have also, if $\left(2^{m}\right)$ denotes a non-alien composition of $2^{m}$,

$$
E^{2^{m-1}}\left(2^{m}\right)=\left(2^{m-1}\right)^{2}, \quad E^{2^{m-2}} E^{2^{m-1}}\left(2^{m}\right)=\left(2^{m-2}\right)^{2^{2}},
$$

and generally

$$
\begin{aligned}
& E^{k}\left(2^{m}\right)=\left(2^{r}\right)^{2^{m-r}}, \\
& \sum_{r}^{m-1} 2^{\mu}<k<\sum_{r-1}^{m-1} 2^{\mu},
\end{aligned}
$$

i.e., if

$$
2^{r}>2^{m}-1-k>2^{r-1}-1 \text {. }
$$

We have then

$$
\begin{gathered}
E^{-k} R\left(\bmod \frac{1}{2} N\right)=\left(2^{r}\right)^{m-1-r-1} \\
2^{r-1}-1<k<2^{r} \\
E^{-2^{m-2}-1} R\left(\bmod \frac{1}{2} N\right)=\left(2^{m-1}\right)^{\lambda}
\end{gathered}
$$

if

and

which is improper. Hence

$A$ regular composition of $2^{m} \lambda$ can be involved $2^{m-1}-1$ times until it becomes improper, and the number of compositions associated with each regular composition is $1+\sum_{0}^{2^{m-1}-2} 2^{\mu}=2^{2^{m-1}-1}$.

Note that the theorem proved in $\$ 35$ is true even for degenerate compositions. Let $P$ be an improper composition of $2^{m} \lambda$ which degenerates to an irreducible composition of $2^{m-1} \lambda$. Then $E^{2^{m-1}-1} P$ is regular and $E^{2^{m-1}} P$ is an improper composition of $2^{m-1} \lambda$ repeated. Hence $E^{2^{m-1}-1} \cdot E^{2^{m-1}} P$ is a pure composition of $2^{m-1} \lambda$ repeated, i.e., $E^{2^{m}-1} P$ is pure and $E^{2^{m}-2} P$ is impure. Similarly, if $P$ is an improper composition of $2^{m} \lambda$ which degenerates to an irreducible composition of $2^{m-r} \lambda$, the number of the first pure evolute is

$2^{m-r}-1+2^{m-r}+2^{m-r+1}+\ldots+2^{m-2}+2^{m-1}=2^{m-r}-1+2^{m-r}\left(2^{r}-1\right)=2^{m}-1$.

38. Generating compositions of a period.

We have seen that, if $P$ belongs to a self-conjugate period, there is 
1908.] Certain periodic Properties of Cyclic compositions of numbers. 299

one member $A$ of the period such that $D A=A . A$ will be cilled the generating composition of the period.

(i.) To find the generating composition of a self-conjugate period.

Let $B$ be any member of the period and suppose $D B=E^{r} B$. Let $A$ be the generating composition, so that $D A=A$, and let $B=E^{\prime \prime} . A$. Let $n$ be the number in the period.

Then

$$
\begin{aligned}
D B & =D E^{\prime \prime} A=E^{24} D A=E^{2 \eta} A \\
& =E^{r} B=E^{r+\imath} A .
\end{aligned}
$$

Therefore

$$
q \equiv r \quad(\bmod n) .
$$

Hence

$$
A=E^{n-r} B \text {. }
$$

This holds whether $N$ is odd or even.

(ii.) Let $N$ be odd, and let $t$ be the smallest index for which

$$
2^{t} \equiv \pm 1 \quad(\bmod N)
$$

Then $D^{t} P=P$ or $P^{\prime}$. Let $s$ be the smallest index for which $D^{s} P$ or its enantiomorph belongs to the period of $P$. Then

$$
D^{s} E^{r} P=E^{r \cdot 2^{s}} D^{s} P \text {. }
$$

Hence, if $Q$ is any member of the period of $P, D^{s} Q$ or its enantiomorph belongs to the period of $P$. Thus $P$ belongs to a conjugate set of $s$ or $2 s$, and $s$ is a factor of $t$.

Suppose there is a member $A$ such that $D^{s} A=A$ or $A^{\prime}$. Take any other member $B$. Let $D^{s} B=E^{\prime} B$ or $E^{r} B^{\prime}$ and $B=E^{\prime \prime} A$.

Then

$$
\begin{aligned}
& D^{s} B=D^{s} E^{\prime \prime} A=E^{\prime \cdot 2^{8}} D^{s} A=E^{\prime \cdot 2^{k}} A \text { or } E^{\prime \prime \cdot 2^{s}} A^{\prime} \\
& =E^{r} B \quad \text { or } E^{r} B^{\prime} \\
& =E^{\prime \prime+r} A \text { or } E^{\prime \prime+r} A^{\prime} \text {. }
\end{aligned}
$$

Hence

$$
q\left(2^{8}-1\right) \equiv r \quad(\bmod u)
$$

If $g$ is the greatest common measure of $2^{s}-1$ and $n$, the solution of this congruence gives $g$ values for $q$. In particular, if the period is normal, $n=2^{t}-1$, and $s$ is a factor of $t$, and in this case $q$ has $2^{s}-1$ values.

The compositions $A=E^{-4} B$ will be called the generating compositions of the period. If $s=t$ every member of the period is a generating composition.

(iii.) Suppose next that $N=2^{m} \lambda$, where $\lambda$ is odd.

$D A$ has all its parts divisible by $2^{s+1}$ (say, $D A$ is divisible by $2^{s+1}$ ) if $A$ is divisible by $2^{s}$, provided $s<m$. Hence $D^{s} A$ cannot $=A$ or $A^{\prime}$ unless $A$ is divisible by $2^{m}$. Then $A=2^{m} A_{1}$, where $A_{1}$ is a composition of $\lambda$. Then, if $D^{s} A_{1}=A_{1}$ or $A_{1}^{\prime}, D^{s} A=A$ or $A^{\prime}$. Corresponding to 
a set of $s$ periods of compositions of $\lambda$, we have, therefore, a set of $s$ periods of compositions of $2^{m} \lambda$, and in these periods every $2^{m}$-th member is divisible by $2^{m}$.

Consider now a period in which there are compositions divisible by $2^{r}$ but none divisible by $2^{r+1}$. Let $A$ be such a composition. Then $D^{n-r} A$ is divisible by $2^{m}$, and, provided $D^{p} A$ is not alien for any value of $p$, $D^{m-r} A$ belongs to a period of a set. (It may happen that $D^{\prime \prime} A$ is divisible by $2^{m}$ where $p<m-r$.)

The periods of $2^{m} \lambda$ are therefore of the following kinds :-

(1) Belonging to a set. This happens if there are compositions in the period divisible by $2^{m}$.

(2) Such that $D^{p} A$ belongs to a set.

(3) Such that $D^{p} A$ is an alien appertaining to $2^{m-1} \lambda$.

In these cases it is always possible to find a generating composition $A$ such that

(1) $D^{\prime} A=A$ or $A^{\prime}$.

(2) $D^{p} A=B$, where $B$ is a generating composition.

(3) $D^{p} A=B B$, where $B$ is a generating composition of $2^{m-1} \lambda$.

To find such a composition.

(1) Let $D^{s} B$ or its enantiomorph $=E^{r} B$, and suppose $B=E^{\prime \prime} A$, where $D^{s} A$ or its enantiomorph $=A$. Then

$$
D^{s} B=E^{u\left(2^{s}-1\right)} B=E^{r} B .
$$

Hence

$$
q\left(2^{s}-1\right) \equiv r \quad(\bmod n) .
$$

If the period is normal, $n=2^{m}\left(2^{t}-1\right)$, and $q$ is determined as one of $2^{2}-1$ values.

(2) Let $D^{p} B$ or its enantiomorph $=E^{r} A$, where $D^{s} A=A$ or $A^{\prime}$, and let

$$
D^{p} E^{q} B=A=E^{q \cdot 2^{p}} D^{p} B=E^{q .2^{p}+r} A .
$$

Hence

$$
q \cdot 2^{p} \equiv-r \quad(\bmod n) .
$$

$r$ must be divisible by $2^{p}$, and $q$ has $2^{p}$ values.

(3) Let $D^{p} B$ or its enantiomorph $=E^{r} A \cdot E^{r} A$, where $D^{s} A$ or its enantiomorph $=A$, and let

$$
\begin{aligned}
D^{p} E^{q} B & =A A=E^{\mu \cdot .^{p}} D^{p} B \\
& =E^{\mu \cdot 2^{p}}\left(E^{r} A \cdot E^{r} A\right)=E^{q \cdot 2^{p}+r} A \cdot E^{q \cdot 2^{2}+r} A .
\end{aligned}
$$

Hence

$$
q \cdot 2^{p} \equiv-r \quad(\bmod n)
$$

and $q$ is determined as before. 
1908.] Certain PERIOdic PRoperties of CYCLIC COMPOSITIONS OF NUMibers. 301

$$
\text { II. }
$$

\section{Enumeration of Cyclic Compositions.}

39. The number of compositions of a number $N$ with $R$ parts has been investigated by MacMahon.* He finds

$$
C(N, R)=\frac{(N-1) !}{(R-1) !(N-R) !}=\left(\begin{array}{l}
N-1 \\
R-1
\end{array}\right) \cdot+
$$

$\mathrm{He}$ also investigates the number of self-inverse compositions, i.e., those which read the same backwards and forwards, and finds

$$
S I C(2 n, 2 r)=S I C(2 n, 2 r-1)=\operatorname{SIC}(2 n-1,2 r-1)=\left(\begin{array}{l}
n-1 \\
r-1
\end{array}\right),
$$

while

$$
S I C(2 n-1,2 r)=0 .
$$

Here we require the number of cyclic compositions of a similar nature.

We shall use the notation

$$
C y(N, R)
$$

for the number of cyclic compositions of $N$ with $R$ parts, $C y$ ( $N$, even) for the number of cyclic compositions with an even number of parts, and $C y(N)$ for the total number of cyclic compositions of $N$; and when we wish to describe more particularly the nature of the compositions we shall use the following abbreviations :-

$$
\begin{array}{ll}
\sigma=\text { symmetrical }, & \alpha=\text { alien } \\
\rho=\text { regular }, & \delta=\text { degenerate, } \\
\pi=\text { pure }, & \iota=\text { improper },
\end{array}
$$

and introduce them into the formula thus

$$
\operatorname{Cy\sigma }(N, R)
$$

denoting the number of symmetrical cyclic compositions of $N$ with $R$ parts.

A bar over a symbol, standing for a minue, denotes the negative, as $\bar{\sigma}=$ not symmetrical, $\bar{\delta}=$ irreducible. Also the product of two symbols will denote the logical product, as $\sigma \rho=$ both symmetrical and regular, $\bar{\alpha} \bar{\rho}=$ neither alien nor regular; and the sum of two symbols will denote their logical sum, as $a+\rho=$ either alien or regular.

* "Memoir on the Theory of the Compositions of Numbers," Plill. Trans., Vol. crxxxiv., A (1893), pp. 835-901. This and a second memoir, Phil. Trans., Vol. ccvir., A (1908). pp. 65-134, deal mainly with compositions of multipartite numbers.

+ Employing the usual continental notation, equivalent to $x-1 C_{k-1}$. 
40. To tind $C y \sigma(N, R)$.

Take any symmetrical cyclic composition of $N$ with $R$ parts.

If $R$ is odd one part occurs singly, the others in pairs.

If $R$ is even and $N$ is odd two parts (whose sum is odd) occur singly, and in the cyclical order these are opposite one another.

If $R$ is even and $N$ is even there may be two parts (whose sum is even) occurring singly, or they may all occur in pairs.

If we take any symmetrical cyclic composition of $2 n+1$ with $2 r+1$ parts, and increase the single part by unity, we get a symmetrical cyclic composition of $2 n+2$ with $2 r+1$ parts, and if we diminish by unity the single part (which is even) in a symmetrical cyclic composition of $2 n+2$ with $2 r+1$ parts, we get a symmetrical cyclic composition of $2 n+1$ with $2 r+1$ parts. Hence

$$
C y \sigma(2 n+1,2 r+1)=C y \sigma(2 n+2,2 r+1) .
$$

Again, if we take any symmetrical cyclic composition of $2 n+1$ with $2 r+1$ parts, and make the pair which is opposite to the single odd part coalesce, we get a symmetrical cyclic composition of $2 n+1$ with $2 r$ parts, and if re break up into a pair the eren part which occurs singly in a symmetrical cyclic composition of $2 n+1$ with $2 r$ parts, we get a symmetrical cyclic composition of $2 n+1$ with $2 r+1$ parts. Hence

$$
C_{y \sigma}(2 n+1,2 r+1)=C_{y \sigma}(2 n+1,2 r) .
$$

Sext take any symmetrical cyclic composition of $2 n+i$ with $2 r$ parts. There are two parts, whose sum is odd, occurring singly. If we diminish the greater of these by unity we get a symmetrical cyclic composition of $2 n$ with $2 r$ parts in which two parts occur singly. But these compositions are not all different. Consider the cyclic composition

$$
k a b c \ldots k+1 \ldots c b a \text {. }
$$

Subtracting 1 from $k+1$, we get

$$
k a b c \ldots k \ldots c b a \text {. }
$$

But this is the same as $\quad k$...cbatiabc...,

and these have been reckoned distinct unless

$$
a b c \ldots
$$

is a self-inverse composition. But in this case if $r$ is even, so that $a b c \ldots$ has a middle part $l$, the composition is cyclically symmetrical about $l$, and would therefore arise also from

$$
k a b c \ldots l \ldots c b a k a b c \ldots l+1 \ldots c b a \text {. }
$$


1908.] Certain Periodic properties of CyClic compositions of nunibers. 303

Again, if $k=l$, and the composition lying between $k$ and $l$ is self-inverse, and has a middle part $m$, the composition will be cyclically symmetrical also about this part, and so on ; but we see that on any supposition there are only two different numbers about which the composition can be symmetrical, viz., $k$ and $l$ if they are different numbers, and $k$ or $l$ and $m$ if $k=l$. Hence

(1) If $r$ is odd $=2 s-1$, and $n=2 m$ or $2 m-1$, the number of cyclic compositions of $2 n$ which are given twice is

$$
\begin{aligned}
& \frac{1}{2} \sum_{k=1}^{n-r+1}\{C(n-k, r-1)-S I C(n-k, r-1)\} \\
= & \frac{1}{2}\left\{\left(\begin{array}{l}
n-1 \\
r-1
\end{array}\right)-\left(\begin{array}{c}
m-1 \\
s-1
\end{array}\right)\right\} .
\end{aligned}
$$

(2) If $r$ is even $=2 s$, the number given twice is

$$
\frac{1}{2} \sum_{k=1}^{n-r+1} C(n-k, r-1)=\frac{1}{2}\left(\begin{array}{c}
n-1 \\
r-1
\end{array}\right) \text {. }
$$

Further, this process does not give all the cyclic compositions of $2 n$ with $2 r$ parts. There are others in which all the parts occur in pairs, e.g.,

$$
k a b c \ldots . . . c b a k \text {. }
$$

Some of these will have been already included if they can also be considered as symmetrical about a single part. This will happen only if

$$
k a b c \ldots
$$

is a self-inverse composition with a middle part, and this requires that $r$ be odd. Hence the number of compositions not included is

(1) If $r=2 s-1$,

$$
\frac{1}{2}\{C(n, r)-S I C(n, r)\}=\frac{1}{2}\left\{\left(\begin{array}{c}
n-1 \\
r-1
\end{array}\right)-\left(\begin{array}{c}
m-1 \\
s-1
\end{array}\right)\right\} ;
$$

(2) If $r=2 s$,

$$
\frac{1}{2} C(n, r)=\frac{1}{2}\left(\begin{array}{c}
n-1 \\
r-1
\end{array}\right) .
$$

Hence

$$
C y \sigma(2 n, 2 r)=C y \sigma(2 n+1,2 \vartheta) \text {. }
$$

Now to find these numbers consider a symmetrical cyclic composition of $2 n+1$ with $2 r+1$ parts. There is a single odd part which may be $1,3,5, \ldots, 2(n-r)+1$, and the remainder is a self-inverse composition. Hence

$$
C y \sigma(2 n+1,2 r+1)=\sum_{k=0}^{n-r} S I C(2 n-2 k, 2 r)=\left(\begin{array}{l}
n \\
r
\end{array}\right) .
$$


So that, finally,

$$
\begin{aligned}
C y \sigma(2 n+1,2 r+1)= & C y \sigma(2 n+1,2 r) \\
= & C y \sigma(2 n+2,2 r+1)=C y \sigma(2 n, 2 r)=\left(\begin{array}{l}
n \\
r
\end{array}\right) .
\end{aligned}
$$

The total number of symmetrical cyclic compositions can now be found

$$
\begin{aligned}
& C y \sigma(2 n+1, \text { even })=\sum_{r=1}^{n}\left(\begin{array}{l}
n \\
r
\end{array}\right)=2^{n}-1, \\
& C y \sigma(2 n+1, \text { odd })=\sum_{r=0}^{n}\left(\begin{array}{l}
n \\
r
\end{array}\right)=2^{n}, \\
& C y \sigma(2 n, \text { even }) \quad=\sum_{r=1}^{n}\left(\begin{array}{c}
n \\
r
\end{array}\right)=2^{n}-1, \\
& C y \sigma(2 n, \text { odd }) \quad=\sum_{r=0}^{n-1}\left(\begin{array}{c}
n-1 \\
r
\end{array}\right)=2^{n-1},
\end{aligned}
$$

and

$$
\begin{aligned}
& C y \sigma(2 n+1)=2^{n+1}-1, \\
& C y \sigma(2 n)=3.2^{n-1}-1 .
\end{aligned}
$$

These numbers, of course, include aliens.

41. We have next to find $C y(N, R)$.

(1) If $N$ is a prime there are no alien or regular compositions* and every cyclic composition with $R$ parts gives $R$ ordinary compositions. Hence

$$
C y(N, R)=\frac{1}{R}\left(\begin{array}{l}
N-1 \\
R-1
\end{array}\right)=\frac{1}{N}\left(\begin{array}{l}
N \\
R
\end{array}\right),
$$

and

$$
C y(N, R)=C y(N, N-R)=\frac{1}{N}\left(\begin{array}{l}
N \\
R
\end{array}\right) .
$$

Therefore

$$
\begin{aligned}
C y(N, \text { even }) & =C y(N, \text { odd }) \\
& =\frac{1}{2} C y(N)=\frac{1}{2 N} \sum_{R=1}^{N-1}\left(\begin{array}{l}
N \\
R
\end{array}\right) \\
& =\frac{1}{2 N}\left(2^{N}-2\right)=\frac{1}{N}\left(2^{N-1}-1\right)
\end{aligned}
$$

\footnotetext{
- We exclude here the composition with $N$ parts, $1,1,1, \ldots$.
} 
1908.] Certain periodic Properties of CyClic Compositions of Numbers. 305

(2) Suppose now that $N$ is not a prime. Then there may be alien or regular compositions. Consider an alien or regular composition of $R$ parts consisting of $S$ parts repeated, so that $p S=R$. Then, if $K$ is the order of the repeated portion $p K=N$. Hence $p$ is a factor of the highest common factor, $G$, of $R$ and $N$. To every value of $p$ there corresponds a certain number of alien or regular compositions, the compositions being regular, according to the definition, only when $p=2$. Now an alien cyclic composition which appertains to $K$ will only give rise to $S$ different compositions by cyclical permutation. Hence

$$
C y \bar{\alpha} \bar{\rho}(N, R)=\frac{1}{R}\{C(N, R)-\Sigma S . C y \bar{\alpha} \bar{\rho}(K, S)\},
$$

where $K=\frac{N}{p}, S=\frac{R}{p}$, and the summation is extended to all values of $p$ which are factors of $A, 1$ being excluded. This gives

$$
C y \bar{\alpha} \bar{\rho}(N, R)=\frac{1}{N}\left(\begin{array}{l}
N \\
R
\end{array}\right)-\Sigma \frac{1}{p} C_{y} \bar{\alpha} \bar{\rho}\left(\frac{N}{p}, \frac{R}{p}\right),
$$

Also $\quad C y \bar{\alpha} \bar{\rho}(N, N-R)=\frac{1}{N}\left(\begin{array}{c}N \\ N-R\end{array}\right)-\Sigma \frac{1}{p} C_{y} \bar{\alpha} \bar{\rho}\left(\frac{N}{p}, \frac{N-R}{p}\right)$.

Since the H.C.F. of $N$ and $N-K$ is the same as that of $N$ and $R$, and if we assume that

$$
C y \bar{\alpha} \bar{\rho}\left(\frac{N}{p}, \frac{N-R}{p}\right)=C y \bar{\alpha} \bar{\rho}\left(\frac{N}{p}, \frac{R}{p}\right),
$$

for all values of $p$, we have

$$
C y \bar{\alpha} \bar{\rho}(N, N-R)=C y \bar{\alpha} \bar{\rho}(N, R) .
$$

But this is true when $N$ is a prime, therefore it is true generally.

(3) If $N$ is odd there are no regular compositions, and

$$
C y \bar{\alpha}(N, N-R)=C y \bar{\alpha}(N, R) .
$$

Also

$$
C y \bar{\alpha}(N, \text { even })=C y \bar{\alpha}(N, \text { odd })=\frac{1}{2} C y \bar{a}(N) .
$$

(4) If $N$ is a power of $2=2^{m}$, there are no aliens with an odd number of parts, and

$$
C y\left(2^{m}, R\right)=\frac{1}{2^{m}}\left(\begin{array}{l}
2^{m} \\
R
\end{array}\right),
$$

8ER. 2. VOL. 7. No. 1024 . 
if $R$ is odd. Hence

$$
\begin{aligned}
C y\left(2^{m}, \text { odd }\right) & =\frac{1}{N}\left\{\left(\begin{array}{c}
N \\
1
\end{array}\right)+\left(\begin{array}{c}
N \\
3
\end{array}\right)+\ldots+\left(\begin{array}{c}
N \\
N-1
\end{array}\right)\right\} \\
& =\frac{1}{N}\left\{1+\left(\begin{array}{c}
N-1 \\
1
\end{array}\right)+\left(\begin{array}{c}
N-1 \\
2
\end{array}\right)+\left(\begin{array}{c}
N-1 \\
3
\end{array}\right)+\ldots\right. \\
& \left.+\left(\begin{array}{l}
N-1 \\
N-2
\end{array}\right)+\left(\begin{array}{l}
N-1 \\
N-1
\end{array}\right)\right\} \\
& =\frac{1}{N} 2^{N-1}=2^{2^{m}-m-1} .
\end{aligned}
$$

42. Number of alien and regular compositions.

$$
C y(a+\rho)(N, R)=\Sigma C y \bar{\alpha} \bar{\rho}\left(\frac{N}{p}, \frac{R}{p}\right),
$$

and

$$
C y \rho(N, 4 r+2)=C y \bar{\alpha}\left(\frac{N}{2}, 2 r+1\right)
$$

hence

$$
C y \rho(N)=C y \bar{\alpha}\left(\frac{N}{2}, \text { odd }\right) .
$$

48. Number of improper compositions.

(1) If $N$ is divisible by 4, every improper composition of $N$ has two involutes which are regular compositions of $2 N$, therefore the number of improper compositions of $N$ is

$$
\begin{aligned}
C y_{\iota}(N, \text { even }) & =\frac{1}{2} C y \rho(2 N) \\
& =\frac{1}{2} C y \bar{\alpha}(N, \text { odd }) .
\end{aligned}
$$

(2) If $N$ is odd, every composition is improper and gives rise to only one regular composition of $2 N$.

(3) If $N=2 \lambda$, where $\lambda$ is odd, the regular compositions are improper and these give rise to only one regular composition of $2 N$. Hence

$$
\begin{aligned}
2 C y \iota \bar{\rho}(N, \text { even })+C y \rho(N) & =C y \rho(2 N)=C y \bar{\alpha}(N, \text { odd }) \\
& =C y \bar{\alpha}(N, \text { even }),
\end{aligned}
$$

where the last expression includes the regular compositions (see next 
1908.] Certain Periodic PRoperties of CyClic Conpositions of Nujibers. 307

section); therefore

$$
\begin{aligned}
\operatorname{Cy} \overline{\bar{\rho}}(N, \text { even }) & =\frac{1}{2} C y \bar{\alpha} \bar{\rho}(N, \text { even }) \\
& =\frac{1}{2} C y \bar{\delta}(N, \text { even }),
\end{aligned}
$$

since, when $N=2 \lambda$, the only compositions which are degenerate are aliens or regular compositions.

44. To prove that $C y \bar{\alpha}(N$, even $)=C y \bar{\alpha}(N$, odd $)$,

the reguiar compositions being included in the left-hand side.

When $N$ is odd, this equation has been already proved, $\$ 41(3)$, for there are no regular compositions. When $N$ is even we have to prove

$$
C y \bar{\alpha}(N, \text { odd })-C y \bar{\alpha} \bar{\rho}(N, \text { even })=C y \rho\left(N^{r}\right) .
$$

Assume this to be true for all factors of $N$. We have, $\$+1 .(2)$,

$$
C y \bar{\alpha}(N, \text { odd })=\frac{1}{N} \sum_{r=0}^{i N-1}\left(\begin{array}{c}
N \\
2 r+1
\end{array}\right)-\Sigma \Sigma \frac{1}{p} C_{y} \bar{a} \bar{\rho}(K, S),
$$

where

$$
\frac{N}{K} \risingdotseq p=\frac{2 r+1}{S} \text {; }
$$

and

$$
C y \bar{\alpha} \bar{\rho}(N, \text { even })=\frac{1}{N} \sum_{r=1}^{j N^{N-1}}\left(\begin{array}{l}
N \\
2 r
\end{array}\right)-\Sigma \Sigma \frac{1}{p^{\prime}} C y \bar{\alpha} \bar{\rho}\left(K^{\prime}, S^{\prime}\right),
$$

where

$$
\frac{N}{K^{\prime}}=p^{\prime}=\frac{2 r}{S^{\prime}}
$$

and, $\$ 42$,

$$
C y \rho(N)=C y \bar{u}\left(\frac{N}{2}, \text { odd }\right) \text {. }
$$

Let $N=2^{m} \lambda$, where $\lambda$ is odd. Then $p$ must be odd and can have all values which are factors of $\lambda, 1$ being excluded, and $S$ must be odd. $p^{\prime}$ may have all values which are factors of $N, 1$ and $N$ being excluded, but if $p^{\prime}$ is odd, $S^{\prime}$ must be even.

$$
\text { Hence } \quad \Sigma \Sigma \frac{1}{p} C_{y} \bar{\alpha} \bar{\rho}(K, S)=\Sigma \frac{1}{p_{1}} C_{y j} \bar{\alpha}(K, \text { odd }),
$$

and $\quad \Sigma \Sigma \frac{1}{p^{\prime}} C y \bar{\alpha} \dot{\bar{\rho}}\left(K^{\prime} . S^{\prime}\right)=\Sigma \frac{1}{p_{1}} C y \bar{\alpha} \bar{\rho}(K$, even $)+\Sigma \frac{1}{p_{2}} C y \bar{\alpha} \bar{\rho}\left(K^{\prime}\right)$,

where $p_{1}$ is odd and $p_{2}$ is even. 
Now $\quad C y \bar{u}(N$, odd $)-C y \bar{\alpha}(N$, even $)$

$=\frac{1}{N} i_{r=0}^{S N-1}\left(\begin{array}{c}N \\ 2 r+1\end{array}\right)-\sum_{r=1}^{N N-1}\left(\begin{array}{l}N \\ 2 r\end{array}\right){ }_{i}^{\prime}+\sum \frac{1}{p_{1}} C y \bar{a} \bar{\rho}\left(K_{1}\right.$, even $)$

$$
+\Sigma \frac{1}{p_{2}} C y \bar{u} \bar{\rho}\left(K_{2}\right)-\Sigma \frac{1}{p_{1}} C y \bar{\alpha}\left(K_{1}, \text { odd }\right)-C y \bar{\alpha}\left(\frac{N}{2}, \text { odd }\right)
$$

$=\frac{2}{N^{N}}+\sum_{a=1} \sum_{\eta=1}^{m} \frac{1}{a .2^{\prime \prime}} C_{y} \bar{a} \bar{\rho}\left(\frac{N}{a .2^{\prime \prime}}\right)+\sum_{i=1}^{m-1} \frac{1}{\lambda .2^{\eta}} C y \bar{a} \bar{\rho}\left(\frac{N^{r}}{\lambda .2^{\prime \prime}}\right)$

$-\sum \frac{1}{p_{1}} C y \rho\left(K_{1}\right)-C y \bar{a}\left(\frac{N}{2}\right.$, odd $)$ (by original assumption)

$=\frac{2}{N}+\sum_{a=1} \cdot \sum_{i=1}^{m} \frac{1}{a \cdot 2^{\prime \prime}} C_{y j} \bar{\alpha} \bar{\rho}\left(\frac{N}{a \cdot 2^{\prime \prime}}\right)+\sum_{y=1}^{m-1} \frac{1}{\lambda \cdot 2^{q}} C_{y} \bar{u} \bar{\rho}\left(\frac{N}{\lambda .2^{i}}\right)$

$$
-\sum_{a=1} \frac{1}{a} C_{y} \bar{a}\left(\frac{N}{2 a}, \text { odd }\right)-\frac{1}{\lambda} C_{y} \bar{a}\left(\frac{N}{2 \lambda}, \text { odd }\right),
$$

where $a$ is any factor of $\lambda$, excluding $\lambda$, but including 1 .

Now

$$
\begin{aligned}
& \frac{1}{a \cdot 2^{\prime-1}} C y \bar{u}\left(\frac{N}{a \cdot 2^{\prime \prime}}, \text { odd }\right)-\frac{1}{a \cdot 2^{\prime}} C y \bar{u} \bar{\rho}\left(\frac{N}{a \cdot 2^{\prime \prime}}\right) \\
= & \left.\frac{1}{a \cdot 2^{\prime \prime}} ; C y \bar{a}\left(\frac{N}{a \cdot 2^{2}}, \text { odd }\right)-C y \bar{\alpha} \bar{\rho}\left(\frac{N}{a \cdot 2^{2}}, \text { even }\right)\right) \\
= & \frac{1}{a \cdot 2^{\prime \prime}} C y \rho\left(\frac{N}{a \cdot 2^{\prime \prime}}\right) \quad \text { (by original assumption) } \\
= & \frac{1}{a \cdot 2^{\prime \prime}} C y \bar{a}\left(\frac{N}{a \cdot 2^{\prime+1}}, \text { odd }\right),
\end{aligned}
$$

provided $q<m$.

Hence, by applying this result successively,

$$
\frac{1}{a} C y \bar{a}\left(\frac{N}{2 a} \text {, odd }\right)-\sum_{i=1}^{m} \frac{1}{a \cdot 2^{\prime \prime}} C y \bar{a} \bar{\rho}\left(\frac{N}{a \cdot 2^{\prime \prime}}\right)=\frac{1}{a \cdot 2^{m}} C y \rho\left(\frac{N}{a \cdot 2^{m}}\right)=0 \text {, }
$$

since

$$
\frac{N}{a \cdot 2^{m}} \text { is odd, }
$$

where $a$ is any factor of $\lambda$, including 1 , but excluding $\lambda$. And

$$
\begin{aligned}
\frac{1}{\lambda} C y \bar{\alpha}\left(\frac{N}{2 \lambda}, \text { odd }\right)-\sum_{l=1}^{m-1} \frac{1}{\lambda .2^{\prime \prime}} C y \bar{\alpha} \bar{\rho}\left(\frac{N}{\lambda .2^{\prime \prime}}\right) & =\frac{1}{\lambda .2^{m-1}} C y \rho\left(\frac{N}{\lambda .2^{m-1}}\right) \\
& =\frac{2}{N} C y \rho(2)=\frac{2}{N} .
\end{aligned}
$$


1908.] Certain periodic properties of Cyclic compositions of numbers. 309

Hence

$$
C y \bar{a}(N, \text { odd })-C y \bar{\alpha}(N, \text { even })=0,
$$

with the given assumptions. But this is true for any odd number, hence it is true generally.

When $N$ is a power of 2 the value of $C y \bar{a}(N$, even) can be found at once by this theorem. For

$$
\begin{aligned}
\operatorname{Cy} \bar{a}\left(2^{m}, \text { even }\right) & =C y\left(2^{m}, \text { odd }\right) \\
& =\frac{1}{N} 2^{N-1}=2^{2^{m}-m-1} .
\end{aligned}
$$

45. We can now find the whole number of even compositions which are not aliens.

$$
\begin{aligned}
C y \bar{\alpha}(N, \text { even }) & =C y \bar{\alpha}(N, \text { odd }) \\
& =\frac{1}{N} \sum_{r=0}^{j N-1}\left(\begin{array}{c}
N \\
2 r+1
\end{array}\right)-\Sigma \frac{1}{p} C y \bar{\alpha}(K, \text { odd }) \\
& =\frac{1}{N} 2^{N-1}-\Sigma \frac{1}{p} C y \bar{\alpha}(K, \text { odd }),
\end{aligned}
$$

where $p$ is given all odd values which are factors of $N$, excluding 1 .

This forms a reduction formula by which we can calculate the number of compositions of $N$ when we know the number of compositions of factors of $N$. We may, however, obtain a formula which will be self-contained.

(1) Let $N=2^{m} \lambda$, where $\lambda$ is a prime.

$$
\begin{aligned}
C y \bar{\alpha}(N, \text { even }) & =\frac{1}{N} 2^{N-1}-\frac{1}{\lambda} C y \bar{a}\left(2^{m}, \text { odd }\right) \\
& =\frac{1}{N} 2^{N-1}-\frac{1}{\lambda} \frac{1}{2^{m}} 2^{2^{m-1}-1} \\
& =\frac{1}{N}\left(2^{N-1}-2^{N / \lambda-1}\right) .
\end{aligned}
$$

(2) Let $N=a^{p}$, where $a$ is an odd prime.

Cy $\bar{\alpha}\left(a^{p}\right.$, even $)$

$$
\begin{aligned}
=\frac{1}{a^{p}} 2^{a^{p}-1} & -\frac{1}{a} C y \bar{\alpha}\left(a^{p-1}, \text { odd }\right) \\
& -\frac{1}{a^{2}} C y \bar{\alpha}\left(a^{p-2}, \text { odd }\right)-\ldots-\frac{1}{a^{p-1}} C y \bar{\alpha}(a, \text { odd })-\frac{1}{a^{\prime \prime}}
\end{aligned}
$$

and $\quad C y \bar{\alpha}\left(a^{p-1}\right.$, odd $)=\frac{1}{a^{p-1}} 2^{a^{p-1}-1}-\frac{1}{a} C y \bar{\alpha}\left(a^{p-2}\right.$, odd $)-\ldots$

$$
-\frac{1}{a^{p-2}} C y \bar{a}(a, \text { odd })-\frac{1}{a^{p-1}} ;
$$




\section{therefore}

$$
\begin{aligned}
& C y \bar{\alpha}\left(a^{p}, \text { even }\right)-\frac{1}{a} C y \bar{\alpha}\left(a^{p-1}, \text { odd }\right) \\
& \quad=\frac{1}{a^{p}} 2^{a^{p}-1}-\frac{1}{a} C y \bar{\alpha}\left(a^{p-1}, \text { odd }\right)-\frac{1}{a^{p}} 2^{a^{p-1}-1} ;
\end{aligned}
$$

hence $\quad C y \bar{\alpha}\left(a^{p}\right.$, even $)=\frac{1}{a^{p}}\left(2^{a^{p}-1}-2^{a^{p-1}-1}\right)=\frac{1}{N}\left(2^{N-1}-2^{N / \alpha-1}\right)$.

(3) Let $N=2^{m} a b$, where $a, b$ are different odd primes.

$$
\begin{aligned}
C y \bar{\alpha}(N, \text { even })=\frac{1}{N} 2^{N-1}-\frac{1}{a} C y \bar{\alpha}\left(2^{m} b, \text { odd }\right)-\frac{1}{b} C y \bar{\alpha}\left(2^{m} a, \text { odd }\right) \\
\quad-\frac{1}{a b} C y \bar{\alpha}\left(2^{m}, \text { odd }\right) \\
=\frac{1}{N} 2^{N-1}-\frac{1}{a} \frac{1}{2^{m} b}\left(2^{2^{m} b-1}-2^{2^{m}-1}\right) \\
\quad-\frac{1}{b} \frac{1}{2^{m} a}\left(2^{2^{m} a-1}-2^{2^{m}-1}\right)-\frac{1}{a b} 2^{2^{m}-1} \\
=\frac{1}{N}\left(2^{N-1}-2^{N / n-1}-2^{N / l-1}+2^{N / a b-1}\right) .
\end{aligned}
$$

It can now be proved that, if $N=2^{m} \cdot a^{a} b^{\beta} c^{\gamma} \ldots$, where $a, b, c, \ldots$ are different odd primes,

$C y \bar{\alpha}(N$, even $)=\frac{1}{N}\left(2^{N-1}-2^{N / a-1}-2^{N / b-1}-\ldots+2^{N / a b-1}+\ldots-2^{N / a b c-1}-\ldots\right)$.

We may write this symbolically $\frac{1}{2 N}\left[2_{\zeta}\right]^{\phi(N)}$,

where $\quad \phi(N)=N\left(1-\frac{1}{a}\right)\left(1-\frac{1}{b}\right) \ldots$, and $\left[x_{5}\right]^{a \pm b}=x^{a} \pm x^{b}$.

Then, to find the number of irreducible compositions, we have to subtract the number of degenerate compositions. Each regular composition gives, including itself, $2^{2^{m-1}-1}$ degenerate compositions, $\S 37$, hence

$$
\begin{aligned}
C y \delta\left(2^{m} \lambda, \text { even }\right) & =2^{2^{m-1}-1} C y \bar{\alpha}\left(2^{m-1} \lambda, \text { odd }\right) \\
& =2^{2^{m-1}-1} \frac{1}{2^{m} \lambda}\left[2_{\zeta}\right]^{\Phi\left(2^{m-1} \lambda\right)},
\end{aligned}
$$

and

$$
C y \bar{\delta}\left(2^{m} \lambda \text {, even }\right)=\frac{1}{2 N}\left\{\left[2_{\zeta}\right]^{\phi(N)}-2^{i N / \lambda}[2]^{\phi(3) N)}\right\} .
$$

The appendix which follows contains a mass of numerical results relating to the number of compositions and the periods for values of $N$ up to 20 . 
1908.] Certain PeRIodic Properties of CYCLIC COMPOSITIONS OF NUMBers. 311

Appendix.

I. Number of Cyclic Compositions and Periods.

\begin{tabular}{|c|c|c|c|c|c|c|c|c|c|}
\hline$N$ & $\begin{array}{l}\widehat{E} \\
0 \\
0 \\
\vdots \\
\frac{3}{15} \\
0\end{array}$ & 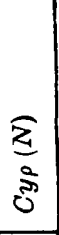 & 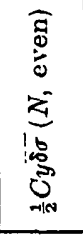 & 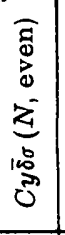 & 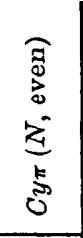 & 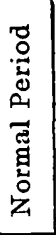 & 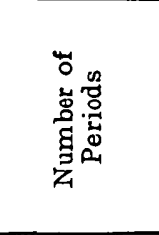 & 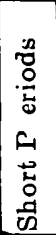 & 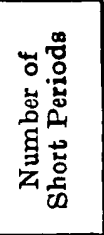 \\
\hline 2 & 1 & 1 & - & - & - & - & - & & \\
\hline 3 & 1 & - & - & 1 & 1 & 1 & 1 & & \\
\hline 4 & 2 & 1 & - & - & - & - & - & & \\
\hline 5 & 3 & - & - & 3 & 3 & 3 & 1 & & \\
\hline 6 & 5 & 1 & - & 4 & 2 & 2 & 1 & & \\
\hline 7 & 9 & - & 1 & 7 & 9 & 7 & 1 & 1 & 2.1 \\
\hline 8 & 16 & 2 & - & - & - & - & - & & \\
\hline 9 & 28 & - & 7 & 14 & 28 & 7 & $2+2.1$ & & \\
\hline 10 & 51 & 3 & 12 & 24 & 24 & 6 & $2+2.1$ & & \\
\hline 11 & 93 & - & 31 & 31 & 93 & 31 & $1+2.1$ & & \\
\hline 12 & 170 & 5 & 56 & 48 & 20 & 4 & $3+2.1$ & & \\
\hline 13 & 315 & - & 126 & 63 & 315 & 63 & $1+2.2$ & & \\
\hline 14 & 585 & 9 & 232 & 112 & 288 & 14 & $4+2.8$ & 2 & 2.2 \\
\hline 15 & 1091 & - & 484 & 123 & 1091 & 15 & $8+2.32$ & $\left\{\begin{array}{l}1 \\
3\end{array}\right.$ & $\begin{array}{r}2.1 \\
1+2.1\end{array}$ \\
\hline 16 & 2048 & 16 & - & - & - & - & - & & \\
\hline 17 & 3855 & - & 1800 & 255 & 3855 & 15 & $16+2.120$ & 5 & 3 \\
\hline 18 & 7280 & 28 & 3388 & 476 & 3626 & 14 & $17+2.121$ & & \\
\hline 19 & 13797 & - & 6643 & 511 & 13797 & 511 & $1+2.13$ & & \\
\hline 20 & 26214 & 51 & 12576 & 360 & 3264 & 12 & $20+2.126$ & & \\
\hline
\end{tabular}

II. Generating Compositions of the Periods and Sets of Periods.

The compositions which are here tabulated are the generating compositions of the periods, and they are arranged in conjugate sets. When $N$ is even $D A$ and $D B$ may belong to the same period or they may be identical, even if $A$ and $B$ belong to two different periods. In this case $A$ is chosen so that $D A$ is a generating composition, and $D A$ is given within square brackets. The compositions in italics belong to racemic sets, i.e., sets which contain both enantiomorphs. For example, if $A=1428$, $D A=1284, D^{2} A=2418=A^{\prime}$, and this set really consists of four periods. The indices are symbolic, e.g., $2^{3} 4$ stands for 2224 . 
$N=3 . \quad$ One period of $1:-12$.

$N=5 . \quad$ One period of $3:-1^{3} 2$.

$N=6$. One period of $2:-24$.

$N=7$. One period of $7:-1^{5} 2$.

A pair of periods of $1:-1^{2} 23$.

$N=9 . \quad$ Two periods of $7:-1^{7} 2 ; 36$.

A pair of periods of $7:-123^{2}$.

$N=10$. Two periods of $6:-2^{9} 4 ; 13^{3}\left[2^{3} 4\right]$.

A pair of periods of $6:-1^{2} 2132\left[2^{3} 4\right]$.

$N=11$. One period of $31:-1^{9} 2$.

A pair of periods of $31:-1^{2} 2124$.

$N=12$. Three periods of $4:-48 ; 121323$ [48]; 1353 [2424].

A pair of periods of $4 \cdot-1^{4} 2123$ [48].

$N=13$. One period of $63:-1^{11} 2$.

Two pairs of periods of $63:-1^{4} 2^{2} 1^{3} 2,1^{7} 312$.

$N=14 . \quad 4$ periods of $14:-$

$2^{5} 4 ; 12121232,141323,1^{3} 21512,\left[2^{5} 4\right]$.

8 pairs of periods of 14 :-

$1^{2} 2154,123^{2} 23,1^{3} 362,12^{2} 135,12^{2} 1^{2} 2^{2} 8$, $1^{3} 3^{2} 212,\left[2^{5} 4\right]$.

$1^{2} 93,135^{2},\left[2^{2} 46\right]$.

2 pairs of periods of 2 :-

$2^{2} 46 ; 1^{2} 412^{2} 12$ [2 $\left.2^{2} 46\right]$.

$N=15 . \quad 8$ periods of $15:-$

$1^{13} 2 ; 510 ; 3^{3} 6 ; 81321212$;

312,$69 ; 1^{7} 21212,1^{4} 2121^{4} 2$.

32 pairs of periods of 15 :-

$1248 ; 121326 ; 123^{4} ; 1^{4} 231212$;

$3^{2} 27,1563 ; 1212123^{2}, 13213212$;

$5^{2} 14,235^{2} ; 1428,1284 ; 1^{2} 2173,1^{2} 52^{2} 4$;

$1^{2} 212512,1^{3} 312^{2} 4 ; 1^{3} 212^{2} 12^{2}, 1^{2} 21^{3} 31^{2} 3$;

$1^{6} 2^{2} 1^{3} 2,1^{2} 21^{8} 3$;

$1^{2} 211,2^{2} 47,1347,1^{2} 67$;

$1^{2} 49,2^{2} 83,4^{2} 16,1257$;

$1^{3} 3^{2} 6,1^{2} 2632,2^{2} 13^{2} 4,312513$. 
1908.] Certain Periodic Propertieg of CXCLIC Compositions of numbers. 313

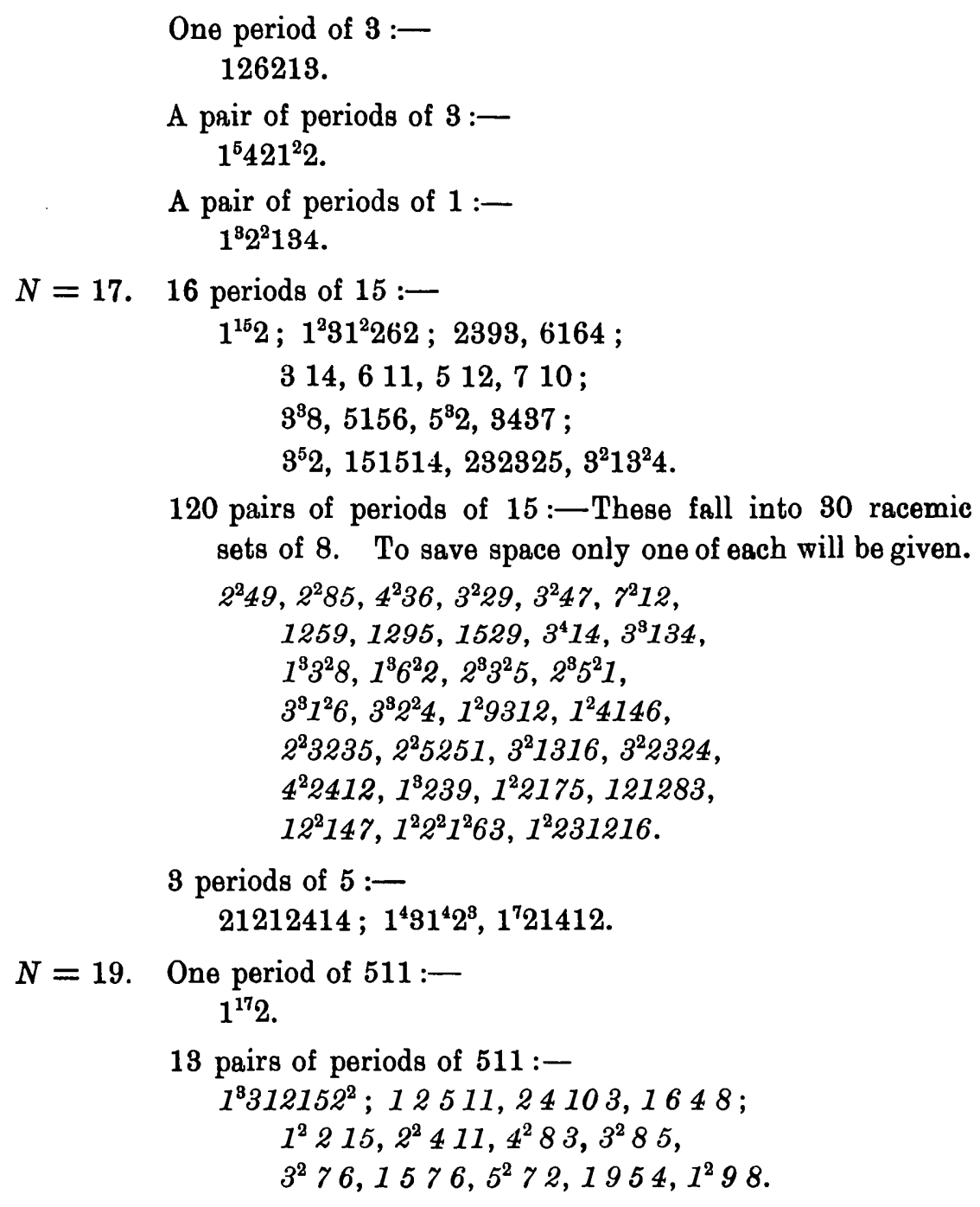

13 pairs of periods of 511 :$1^{8} 312152^{2} ; 12511,24103,1648$;

$$
\begin{aligned}
& 1^{2} 215,2^{2} 411,4^{2} 83,3^{2} 85, \\
& 3^{2} 76,1576,5^{2} 72,1954,1^{2} 98 .
\end{aligned}
$$

